\title{
Revisiting coupling selection rules in heterotic orbifold models
}

\author{
Tatsuo Kobayashi, ${ }^{a}$ Susha L. Parameswaran, ${ }^{b}$ Saúl Ramos-Sánchez ${ }^{c}$ and \\ Ivonne Zavala ${ }^{d}$ \\ ${ }^{a}$ Department of Physics, Kyoto University, \\ Kyoto 606-8502, Japan \\ ${ }^{b}$ Department of Physics and Astronomy, Uppsala University, \\ P.O. Box 803, S-75108, Uppsala, Sweden \\ ${ }^{c}$ Departamento de Física Teórica, Instituto de Física, UNAM, \\ México D.F. 04510, México \\ ${ }^{d}$ Bethe Center for Theoretical Physics and Physikalisches Institut der Universität Bonn, \\ Nussallee 12, 53115 Bonn, Germany \\ E-mail: kobayash@gauge.scphys.kyoto-u.ac.jp, \\ susha.parameswaran@physics.uu.se, ramos@fisica.unam.mx, \\ zavala@th.physik.uni-bonn.de
}

ABSTRACT: We study L-point couplings between twisted sector fields in heterotic orbifold compactifications, using conformal field theory. Selection rules provide an easy way to identify which couplings are non-vanishing. Those used in the current literature are gauge invariance, R-charge conservation and the space group selection rule, but they are not the whole story. We revive and refine a fourth selection rule, due to symmetries in the underlying torus lattice, and introduce a fifth one, due to the existence or not of classical worldsheet instanton solutions to mediate the couplings. We consider briefly the phenomenological consequences of the additional rules, in particular for recent orbifold constructions whose field content correspond to that of the MSSM. The structure of the exotic mass matrices is unaltered and many dimension-5 proton-decay operators vanish.

KEywords: Superstrings and Heterotic Strings, Conformal Field Models in String Theory, Global Symmetries

ARXIV EPRINT: 1107.2137 


\section{Contents}

1 Introduction $\quad 1$

2 Twist fields, vertex operators and correlation functions 3

2.1 The building blocks 3

$\begin{array}{lll}2.2 & \text { The standard selection rules } & 6\end{array}$

3 L-point correlation functions and more selection rules $\quad 8$

3.1 H-momentum conservation 8

3.2 Decomposition into classical and quantum parts 9

$\begin{array}{lll}3.3 & 3 \text {-point couplings } & 12\end{array}$

3.3.1 A new rule: "Rule 5" 12

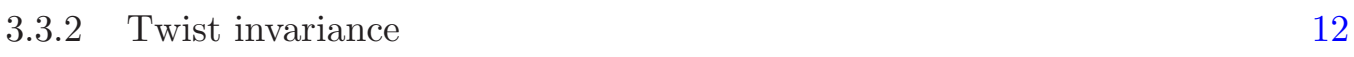

$\begin{array}{ll}\text { 3.3.3 } & \text { Remembering Rule } 4 \\ \end{array}$

$\begin{array}{ll}3.4 & \text { Higher order couplings } \\ \end{array}$

$\begin{array}{llr}3.4 .1 & \text { Rule } 5 & 14\end{array}$

$\begin{array}{lll}3.4 .2 & \text { Rule } 4 & 15\end{array}$

$\begin{array}{lll}3.5 & \text { On effective couplings } & 16\end{array}$

4 A concrete example: the $T^{6} / \mathbb{Z}_{6-I I}$ orbifold $\quad 17$

$\begin{array}{lll}4.13 \text {-point couplings } & 17\end{array}$

$\begin{array}{lll}4.2 & 4 \text {-point couplings } & 18\end{array}$

$\begin{array}{lll}4.3 & \text { General L-point couplings } & 18\end{array}$

4.4 Explicit $\mathbb{Z}_{6-I I}$ MSSM candidates 20

$\begin{array}{lll}5 & \text { Discussion } & 21\end{array}$

\section{Introduction}

After around a quarter of a century of work, heterotic orbifold compactifications continue to provide a promising framework for building realistic models of Nature from string theory. One of the huge advantages of these scenarios is their simplicity. Orbifold compactifications are globally consistent constructions with a clear geometrical interpretation, and thus several phenomena can be understood in an intuitive geometrical way. Moreover, they correspond to free CFTs, and in particular one can solve strings on the orbifold. Hence, many quantities, such as the string couplings, can be computed exactly.

Recently, it has become possible to build orbifold models whose spectrum corresponds to the MSSM with no chiral exotics [1-6]. Also other interesting models have been constructed [7-10]. It is now important to study more closely the phenomenology of these 
models. Among the issues that must be addressed are the decoupling of vector-like exotics, the hierarchy of quark and lepton masses and the suppression of proton decay operators. To tackle these problems, the couplings in the low energy effective field theory are required. The trilinear couplings including ground state twisted fields are well understood [11-16], and some studies including excited twisted states have also been made [11, 17]. Analytic computations of higher order coupling strengths were considered recently in [18]. In general, couplings between twisted strings are mediated by worldsheet instantons, and are exponentially suppressed in the area separating the participating states and wrapped by the instantons $[11,12]$.

Actually, the first question asked when considering couplings in a given model is simply which ones are non-vanishing. The non-vanishing couplings are those allowed by the string theory selection rules. These were discussed from the very beginning in the orbifold literature $[11,12]$, and a comprehensive account of their status in the eighties can be found in $[19,20]$. Aside from the usual constraint of gauge invariance, they may be briefly summarized as follows. (i) (Point and) space group invariance. This comes from the requirement that the boundary conditions of participating strings are such that they can interact. The various twisted sectors in a coupling $\theta^{k_{1}} \theta^{k_{2}} \ldots \theta^{k_{\mathrm{L}}}$ must satisfy $k_{1}+k_{2}+\cdots+k_{\mathrm{L}}=0 \bmod \mathrm{N}$ for a $\mathbb{Z}_{\mathrm{N}}$ orbifold with the twist $\theta$, and the fixed points on which the twisted fields are localized are restricted. (ii) H-momentum conservation. The $\mathrm{SO}(10)$ lattice momentum associated with the bosonized right-moving fermions must be conserved. (iii) Twist invariance. For factorizable orbifolds this means that the couplings are invariant under independent orbifold twists in each of the planes. Thus, the numbers of bosonic oscillators in the corresponding correlation function are restricted. (iv) Rule 4. When all twisted fields are at the same fixed point, the correlation function enjoys not only the twist symmetry but the full torus lattice symmetry, which can include an additional $\mathbb{Z}_{2}$ or $\mathbb{Z}_{3}$ symmetry. In that case, the numbers of bosonic oscillators are restricted further.

These selection rules have evolved since their original formulation. The H-momentum of a given state depends on the ghost-picture with which we choose to write the corresponding vertex operator. The same holds for the number of right-moving bosonic oscillators. Therefore, H-momentum conservation and twist invariance have been incorporated into a picture-independent R-charge conservation, corresponding to a discrete R-symmetry in the low energy effective field theory [8]. Attempts have also been made to understand the stringy space group selection rule in terms of conventional global symmetries in the field theory limit, with partial success $[2,8,21]$. Also, these stringy selection rules can lead to non-Abelian discrete flavor symmetries [8, 22, 23]. Meanwhile, Rule 4, which has also evaded a field theoretic interpretation [20], has been lost in the recent literature. Thus, the current selection rules that have been applied for example in [3-6] are gauge invariance, the space group selection rule and R-charge conservation.

The purpose of the present paper is to readdress the coupling selection rules in heterotic orbifold models in the light of the previous discussion. In principle, the single condition of $\mathrm{R}$-charge conservation is not sufficient to ensure both $\mathrm{H}$-momentum and twist invariance. We explain how R-charge conservation put together with point group invariance does however turn out to be sufficient. We reinstate Rule 4, and study it in some detail. We also 
identify a new selection rule, Rule 5, by which couplings may be forced to be vanishing if the classical instanton solutions that would mediate them are zero. Our focus is on the derivation of the rules by considering the string theory CFT correlation functions, and we leave their interpretation within the low energy effective field theory for future work. By implementing Rules 4 and 5 in concrete models we confirm that they are non-trivial, and begin to explore their phenomenological implications.

The paper is organized as follows. In the next section we briefly review heterotic orbifold constructions and in particular the CFT ingredients necessary to compute couplings. We also outline the standard selection rules. Then we present the general form of the correlation function corresponding to L-point couplings in section 3, and use this to derive H-momentum conservation and twist invariance, as well as Rules 4 and 5. In section 4 we illustrate how the new rules are implemented in the example of a $\mathbb{Z}_{6-I I}$ orbifold, and begin to investigate their impact in explicit MSSM models. Lastly, we close with some further discussion about the new rules.

Comprehensive reviews on orbifold compactifications can be found in [24, 25], and accounts from a more recent perspective are given in [21, 26, 27].

\section{Twist fields, vertex operators and correlation functions}

In this section we introduce the setup of heterotic strings on orbifolds, and the basic ingredients necessary to compute couplings at any order. Having described the untwisted and twisted massless closed string states on the orbifold, we construct the corresponding vertex operators in the conformal field theory. The prescription for computing the couplings is then to calculate the correlation functions of the vertex operators, and integrate over the location of the operators on the world-sheet.

\subsection{The building blocks}

We begin by taking a $6 \mathrm{D}$ torus, which is obtained as $\mathbb{R}^{6} / \Lambda$, where $\Lambda$ is some $6 \mathrm{D}$ lattice that we usually classify using the root lattices of the semi-simple Lie algebras of rank six. To construct the orbifold, we quotient the torus by a finite-order automorphism of $\Lambda$, called the point group. Possible lattices $\Lambda$ to realize $\mathbb{Z}_{\mathrm{N}}$ orbifolds can be found in $[16,28]$. For simplicity we restrict ourselves to the $\mathbb{Z}_{\mathrm{N}}$ orbifolds whose underlying torus lattice can be factorized into three $2 \mathrm{D}$ torii. These so-called factorizable orbifolds are $T^{6} / \mathbb{Z}_{3}, T^{6} / \mathbb{Z}_{4}$, $T^{6} / \mathbb{Z}_{6-I}$ and $T^{6} / \mathbb{Z}_{6-I I}$. The orbifold twist $\theta$ can be represented by the twist vector

$$
v=\frac{1}{\mathrm{~N}}(0,0, a, b, c),
$$

with the conditions $a+b+c=0 \bmod \mathrm{N}$ and $a, b, c \neq 0 \bmod \mathrm{N}$ required by $\mathcal{N}=1$ supersymmetry. We denote the components of the twist vector by $v^{m}$, with $m=1, \ldots 5$. The last three components describe the non-trivial action of the twist on the three internal planes, and we call them $v^{i}$ with $i=1,2,3$.

Heterotic string states on the orbifold are composed of the following elements. There are the bosonic strings on the $4 \mathrm{D}$ spacetime and the $6 \mathrm{D}$ orbifold, their right-moving superpartners and the left-moving gauge parts. There are two kinds of massless closed string 
states on the orbifold: untwisted strings and twisted ones. For the $\theta^{k}$-twisted sector ( $0 \leq k \leq \mathrm{N}-1$ and $k=0$ is the untwisted sector), the boundary conditions for the complexified $6 \mathrm{D}$ string coordinates, $X^{i}=X^{M=2 i-1}+i X^{M=2 i}$, are

$$
X^{i}(\sigma+\pi)=\left(\theta^{k} X\right)^{i}(\sigma)+\lambda^{i}, \quad \lambda^{i} \in \Lambda^{i},
$$

and we have corresponding boundary conditions on the remaining fields. The center of mass of the twisted states are localized at the fixed points or planes $f$ of the orbifold geometry, and we denote the latter with their corresponding space group elements $\left(\theta^{k}, \lambda\right)$. Thanks to the orbifold identifications, the fixed points $\left(\theta^{k}, \lambda\right)$ and $\left(\theta^{k}, \theta^{l} \lambda+\left(1-\theta^{k}\right) \Lambda\right)$ for any integer $l$ belong to the same conjugacy class, and they are physically equivalent. However, the fixed points of $\theta^{k}$ (when $2 \leq k \leq N-2$ ) are not always fixed points of $\theta$, so the conjugacy classes of a higher $\theta^{k}$-twisted sector are not typically in one-to-one correspondence with the fixed points of $\theta^{k}$. In that case, physical states are $\theta$-invariant linear combinations of states located at different fixed points of $\theta^{k}$, which otherwise carry exactly the same quantum numbers $[15,26,27]$.

The physical states in the string Hilbert space we just introduced are equivalent to fields in the orbifold conformal field theory. We define a twist field $\sigma_{(k, f)}(z, \bar{z})$ as the field that creates a ground state in the sector twisted by $\theta^{k}$ (for $1 \leq k \leq \mathrm{N}-1$ ) so: $\left|\sigma_{(k, f)}\right\rangle=$ $\sigma_{(k, f)}(0,0)|0\rangle$. The twist field incorporates the non-trivial boundary condition (2.2) by inducing a non-trivial monodromy; near a twist field located at the origin the field $X^{i}$ undergoes a phase rotation

$$
X^{i}\left(e^{2 \pi i} z, e^{-2 \pi i} \bar{z}\right)=e^{2 \pi i \mathbf{k}^{i}} X^{i}(z, \bar{z}),
$$

where $z=e^{2(\tau+i \sigma)}$. This corresponds to a branch point with order $\mathbf{k}^{i}$ in each plane, where $\mathbf{k}^{i}=k v^{i} \bmod 1$, such that $0<\mathbf{k}^{i}<1$. The operator product expansions (OPEs) for the free fields $\partial X, \bar{\partial} X, \partial \bar{X}$ and $\bar{\partial} \bar{X}$ (where $\partial=\partial / \partial z, \bar{\partial}=\partial / \partial \bar{z}$ ) with the twist fields in each plane are given by [12]

$$
\begin{aligned}
\partial X(z, \bar{z}) \sigma(w, \bar{w}) & \sim(z-w)^{-\left(1-\frac{k}{N}\right)} \tau(w, \bar{w})+\ldots, \\
\partial \bar{X}(z, \bar{z}) \sigma(w, \bar{w}) & \sim(z-w)^{-\frac{k}{N}} \tau^{\prime}(w, \bar{w})+\ldots, \\
\bar{\partial} X(z, \bar{z}) \sigma(w, \bar{w}) & \sim(\bar{z}-\bar{w})^{-\frac{k}{N}} \tilde{\tau}^{\prime}(w, \bar{w})+\ldots, \\
\bar{\partial} \bar{X}(z, \bar{z}) \sigma(w, \bar{w}) & \sim(\bar{z}-\bar{w})^{-\left(1-\frac{k}{N}\right)} \tilde{\tau}(w, \bar{w})+\ldots,
\end{aligned}
$$

where we have written down the most singular parts in the expansion. Also, we have defined four excited twist fields $\tau$, and the tildes denote fields related by complex conjugation on the world-sheet.

Having introduced the twist fields, we can write down the vertex operators for twisted states. For our purposes it is enough to consider the zero $4 \mathrm{D}$ momentum limit. The emission vertex for a twisted bosonic field is then given by ${ }^{1}$

$$
V_{-1}=e^{-\phi} \prod_{i=1}^{3}\left(\partial X^{i}\right)^{\mathcal{N}_{L}^{i}}\left(\partial \bar{X}^{i}\right)^{\overline{\mathcal{N}}_{L}^{i}} e^{i q_{\mathrm{sh}}^{m} H^{m}} e^{i p_{\mathrm{sh}}^{I} X^{I}} \sigma_{(k, f)}^{i},
$$

\footnotetext{
${ }^{1}$ We omit the cocycle factors needed to satisfy fermionic anti-commutation properties [29], which would determine the overall sign of the correlation functions. We also omit twist-dependent normalization factors and factors coming from the definition of the excited twist fields, eq. (2.4).
} 
whilst for a twisted fermionic field it is

$$
V_{-1 / 2}=e^{-\phi / 2} \prod_{i=1}^{3}\left(\partial X^{i}\right)^{\mathcal{N}_{L}^{i}}\left(\partial \bar{X}^{i}\right)^{\overline{\mathcal{N}}_{L}^{i}} e^{i q_{\mathrm{sh}}^{(f) m} H^{m}} e^{i p_{\mathrm{sh}}^{I} X^{I}} \sigma_{(k, f)}^{i} .
$$

In these expressions, $H^{m}$ are the five, free bosonic fields representing the right-moving fermions through bosonization, $X^{I}(I=1, \ldots, 16)$ correspond to the gauge fields, and $\partial X^{i}$ and $\partial \bar{X}^{i}$ denote bosonic oscillators for the left-movers. The number of oscillators creating any excited massless modes are counted by the oscillator numbers, $\mathcal{N}_{L}^{i}$ and $\overline{\mathcal{N}}_{L}^{i}$. The momentum in the gauge part can be written as $p_{\mathrm{sh}}=p+k V+n_{a} W_{a}$, with $p$ a vector in the $E_{8} \times E_{8}$ lattice, $V$ the shift vector that describes the embedding of the twist in the gauge degrees of freedom and $n_{a} W_{a}$ any discrete Wilson lines present. Similarly, we have written the so-called H-momentum carried by the bosonized fermions as $q_{\mathrm{sh}}=q+k v$, where $q$ lies on the $\mathrm{SO}(10)$ (vector) weight lattice and $v$ is the twist vector. The H-momentum in the spinor representation is written as $q_{\mathrm{sh}}^{(f)}$, and is related to that in the vector representation by $q_{\mathrm{sh}}=$ $q_{\mathrm{sh}}^{(f)}+(1,1,1,1,1) / 2$. Below, we will use the fact that the summation of H-charge over the three internal planes is fixed for each vertex operator [30]. In particular, counting positive chiral states, ${ }^{2} V_{-1}$ has summed H-charge +1 , whereas $V_{-1 / 2}$ has summed H-charge $-1 / 2$. We have factored the bosonic twist fields into three $2 \mathrm{D}$ components. The final component of the vertex operators is a free scalar, $\phi$, related to the superconformal ghost system, and the subscript on the vertex operator $V$ indicates the ghost-charge. Lastly, we note that throughout the paper, we suppress an overall normalization in the vertex operators whose dimension is given in terms of the string scale, $\alpha^{\prime}=l_{s}^{2}$, by $\alpha^{\prime 1 / 2} \prod_{i=1}^{3} \alpha^{\prime-\left(\mathcal{N}_{L}^{i}-\overline{\mathcal{N}}_{L}^{i}\right) / 2}[31]$. So with each field in a coupling, we have a suppression by one inverse mass dimension as expected.

Note that untwisted fields with $k=0$ are included in our discussion, with the corresponding vertex operators obtained by taking $\sigma_{(k, f)}^{i}$ to 1 in the expressions above. Untwisted fields are each associated to one of the planes, and we call $U_{1}, U_{2}$ and $U_{3}$, respectively, untwisted sectors with H-momenta $q_{\mathrm{sh}}=(0,0,1,0,0),(0,0,0,1,0)$ and $(0,0,0,0,1)$.

It will be convenient to use the picture-changing formalism, whereby physically equivalent vertex operators carry superconformal ghost-charge that differ by an integer. In particular we will use bosonic twisted vertex operators in the 0-picture, which are given by

$$
\begin{aligned}
V_{0}=\left(\alpha^{\prime} / 2\right)^{\frac{1}{2}} & \sum_{j=1}^{3}\left(e^{i q_{0}^{j m} H^{m}} \bar{\partial} X^{j}+e^{-i q_{0}^{j m} H^{m}} \bar{\partial} \bar{X}^{j}\right) \\
& \times \prod_{i=1}^{3}\left(\partial X^{i}\right)^{\mathcal{N}_{L}^{i}}\left(\partial \bar{X}^{i}\right)^{\overline{\mathcal{N}}_{L}^{i}} e^{i q_{\mathrm{sh}}^{m} H^{m}} e^{i p_{\mathrm{sh}}^{I} X^{I}} \sigma_{(k, f)}^{i} .
\end{aligned}
$$

The picture-changing operation ${ }^{3}$ to the 0 -picture has a contribution to the H-momentum given by $q_{0}^{1}=(0,0,1,0,0), q_{0}^{2}=(0,0,0,1,0)$ and $q_{0}^{3}=(0,0,0,0,1)$, and introduces right-

\footnotetext{
${ }^{2}$ Throughout the paper, we refer to the positive chiral states in the spectrum. For their negative chiral partners, $V_{-1}$ has summed H-charge -1 , and $V_{-1 / 2}$ has summed H-charge $+1 / 2$.

${ }^{3}$ Notice that in our conventions, the fields transform under the orbifold twist as: $X^{I} \rightarrow X^{I}+2 \pi V^{I}$, $H^{m} \rightarrow H^{m}-2 \pi v^{m}, \partial X^{i} \rightarrow e^{i 2 \pi v^{i}} \partial X^{i}, \partial \bar{X}^{i} \rightarrow e^{-i 2 \pi v^{i}} \partial \bar{X}^{i}, \bar{\partial} X^{i} \rightarrow e^{i 2 \pi v^{i}} \bar{\partial} X^{i}$ and $\bar{\partial} \bar{X}^{i} \rightarrow e^{-i 2 \pi v^{i}} \bar{\partial} \bar{X}^{i}$, so the picture changing operator is twist invariant, as it must be.
} 
moving oscillators that will be counted by $\mathcal{N}_{R}^{i}, \overline{\mathcal{N}}_{R}^{i}$. The $V_{0}$ vertex operator is thus a sum of terms, each with a summed H-charge of 2 or 0.

With the vertex operators in hand, we can compute the scattering amplitudes for the massless states and deduce the coupling terms in the $4 \mathrm{D}$ effective field theory. A term $\Phi^{n+3}$ in the superpotential, with $\Phi$ a chiral superfield with components $(\phi, \psi)$, can be inferred most straightforwardly from an interaction of the form $\psi \psi \phi^{n+1}$. Therefore, we want to investigate tree-level ${ }^{4}$ correlation functions of the form

$$
\left\langle V_{-1 / 2}\left(z_{1}, \bar{z}_{1}\right) V_{-1 / 2}\left(z_{2}, \bar{z}_{2}\right) V_{-1}\left(z_{3}, \bar{z}_{3}\right) V_{0}\left(z_{4}, \bar{z}_{4}\right) \ldots V_{0}\left(z_{n+3}, \bar{z}_{n+3}\right)\right\rangle,
$$

where the ghost-charges have been chosen to cancel the background value of 2 on the sphere. Of course, if such a coupling is vanishing, then so must be its supersymmetric relatives, such as the terms $\phi^{n+2} \bar{\phi}^{n+2}$ in the scalar potential that arise from the superpotential.

In the following, we use the index $\alpha=1, \ldots, \mathrm{L}$ to label the states participating in an L-point coupling.

\subsection{The standard selection rules}

We have seen above that vertex operators consist of several parts: the $4 \mathrm{D}$ part (whose momentum we have set to zero), the $6 \mathrm{D}$ twist field, the bosonized fermions, the gauge part and the left and right-moving oscillators. Since we have a free field theory, the correlation functions also factor accordingly, with the parts corresponding to $H^{m}, X^{I}$ and ghosts given by the well-known result similar to the Veneziano amplitude. They can be found for example in $[18,31]$.

Each part of the correlation function has its own selection rule for allowed couplings. The most familiar of these are conservation of $4 \mathrm{D}$ momentum and conservation of the momentum of the gauge part,

$$
\sum_{\alpha=1}^{\mathrm{L}} p_{\operatorname{sh} \alpha}^{I}=0
$$

which is simply the requirement of gauge invariance. These conditions can be derived via the textbook result [31]:

$$
\left\langle\prod_{\alpha=1}^{\mathrm{L}} e^{i p_{\alpha} \cdot X\left(z_{\alpha}\right)}\right\rangle \sim \delta^{(16)}\left(\sum_{\alpha=1}^{\mathrm{L}} p_{\alpha}\right) \prod_{\alpha, \beta=1, \alpha<\beta}^{\mathrm{L}}\left(z_{\alpha}-z_{\beta}\right)^{\alpha^{\prime} p_{\alpha} \cdot p_{\beta} / 2} .
$$

An analogous computation for the bosonized fermions' part of the correlation function implies that the total H-momentum in each plane ${ }^{5}$ must also be conserved in an interaction. For example, for a 3 -point coupling $\left\langle V_{-1 / 2} V_{-1 / 2} V_{-1}\right\rangle$, we can express the rule in terms of the H-momentum of the scalar components as

$$
\sum_{\alpha=1}^{3} q_{\mathrm{sh} \alpha}^{i}=1
$$

\footnotetext{
${ }^{4}$ Recall that, as a result of holomorphicity, the superpotential does not receive corrections in string perturbation theory [32].

${ }^{5}$ Throughout the paper we use the term "summed H-momentum" to refer to the sum of H-momentum over the three complex planes for a given state, and "total H-momentum" to refer to the total H-momentum in a given plane from all states participating in an interaction.
} 
where we have used the relation between the fermionic and bosonic representations $q_{\mathrm{sh}}^{i}=$ $q_{\mathrm{sh}}^{(f) i}+\frac{1}{2}$. For the higher order couplings, we have to take care of the fact that the picturechanging operator gives non-trivial contributions to the H-momentum. We describe in the following section how H-momentum conservation then plays an important role in determining the structure of the corresponding correlation functions. Meanwhile, a pictureindependent conservation law that turns out to incorporate H-momentum conservation can also be constructed, as we discuss below.

Another invariance property of the correlation functions is twist invariance. As we prove in the following section, for the factorizable orbifolds it happens that couplings are invariant under independent orbifold twists in each of the three 2D planes:

$$
\begin{array}{rlrl}
\partial X^{i} & \rightarrow e^{i 2 \pi v^{i}} \partial X^{i}, & \partial \bar{X}^{i} \rightarrow e^{-i 2 \pi v^{i}} \partial \bar{X}^{i}, \\
\bar{\partial} X^{i} \rightarrow e^{i 2 \pi v^{i}} \bar{\partial} X^{i}, & \bar{\partial} \bar{X}^{i} \rightarrow e^{-i 2 \pi v^{i}} \bar{\partial} \bar{X}^{i} .
\end{array}
$$

This corresponds to a constraint on the total oscillator numbers present in a coupling:

$$
\mathcal{N}_{L}^{i}+\mathcal{N}_{R}^{i}-\overline{\mathcal{N}}_{L}^{i}-\overline{\mathcal{N}}_{R}^{i}=0 \bmod \mathrm{N}^{i}
$$

with $\mathrm{N}^{i}$ the orders of the orbifold twist in the 2D plane, i.e. the smallest possible integers such that $\mathrm{N}^{i} v^{i}=0 \bmod 1$ (no summation over $i$ ). Also, here and below, we denote $\mathcal{N}_{L}^{i}=$ $\sum_{\alpha=1}^{\mathrm{L}} \mathcal{N}_{L \alpha}^{i}$ and so on.

Picture-changing, as well as introducing new H-momentum, incorporates new oscillators into the vertex operators and correlation functions. However, for a given state we can define the picture-invariant R-charge $[8,20]$

$$
\mathrm{R}_{\alpha}^{i}=q_{\mathrm{sh} \alpha}^{i}-\mathcal{N}_{L \alpha}^{i}+\overline{\mathcal{N}}_{L \alpha}^{i}
$$

for which the contributions from the additional H-momentum and right-moving oscillators in the picture-changing operator cancel against each other. Then we can define the Rcharge conservation law as

$$
\sum_{\alpha=1}^{\mathrm{L}} \mathrm{R}_{\alpha}^{i}=1 \bmod \mathrm{N}^{i} .
$$

It can be seen as a consequence of combining H-momentum conservation and twist invariance.

The final well-known selection rule arises from the requirement that the boundary conditions of the twisted closed strings are such that they can join together to form other closed strings. This is called the space group selection rule, and takes the form

$$
\prod_{\alpha=1}^{\mathrm{L}}\left[g_{\alpha}\right]=(\mathbb{1}, 0)
$$

where $\left[g_{\alpha}\right]$ represents some element of the conjugacy class of the space group element $g_{\alpha}$. The space group selection rule includes the point group selection rule, which requires 
$\prod_{\alpha=1}^{\mathrm{L}} \theta^{k_{\alpha}}=1$, or $\sum_{\alpha=1}^{\mathrm{L}} k_{\alpha}=0 \bmod \mathrm{N}$ for the $\mathbb{Z}_{\mathrm{N}}$ orbifold. In terms of the explicit space group elements, it can be written as

$$
\begin{aligned}
&\left(1-\theta^{k_{\mathrm{L}}}\right)\left(\theta^{m_{\mathrm{L}}} f_{\mathrm{L}}+\tau_{\mathrm{L}}\right)+\theta^{k_{\mathrm{L}}}\left(1-\theta^{k_{\mathrm{L}-1}}\right)\left(\theta^{m_{\mathrm{L}-1}} f_{\mathrm{L}-1}+\tau_{\mathrm{L}-1}\right)+\ldots \\
& \ldots+\theta^{k_{\mathrm{L}}+k_{\mathrm{L}-1}+\cdots+k_{2}}\left(1-\theta^{k_{1}}\right)\left(\theta^{m_{1}} f_{1}+\tau_{1}\right)=0,
\end{aligned}
$$

for some $\tau_{\alpha} \in \Lambda$ and arbitrary $m_{\alpha} \in \mathbb{Z}$. Thus we see that the space group selection rule restricts the combinations of fixed points that can enter a coupling.

In summary, the selection rules that are applied in the current literature are gauge invariance, R-charge conservation and the space group selection rule. We observe here that, in principle, the R-charge conservation law is not a sufficient condition to ensure that the two constraints of twist invariance and H-momentum conservation are individually respected, but only a necessary one. ${ }^{6}$ However, one can check in explicit models that all the couplings allowed by both point group invariance and R-charge conservation, automatically also satisfy H-momentum conservation. Thus, imposing point group invariance and $\mathrm{R}$ charge conservation is in fact enough to ensure that both the H-momentum and twist invariance conditions are satisfied. We shall discuss this in a little more detail in the following section.

\section{L-point correlation functions and more selection rules}

In the previous section we outlined the basic building blocks necessary to study correlation functions for twisted states in heterotic orbifolds. We now study in more detail the structure of the correlation functions. We assume that the standard selection rules of gauge invariance, R-charge conservation and the space group selection rule have been applied, and identify two further rules that force some couplings to be vanishing.

Our starting point will be the non-trivial part of the general correlation function, which takes the form (we set $\alpha^{\prime}=2$ )

$$
\mathcal{F}=\prod_{i=1}^{3}\left\langle\left(\partial X^{i}\right)^{\mathcal{N}_{L}^{i}}\left(\partial \bar{X}^{i}\right)^{\overline{\mathcal{N}}_{L}^{i}}\left(\bar{\partial} \bar{X}^{i}\right)^{\overline{\mathcal{N}}_{R}^{i}} \sigma_{\left(k_{1}, f_{1}\right)}^{i} \cdots \sigma_{\left(k_{\mathrm{L}}, f_{\mathrm{L}}\right)}^{i}\right\rangle,
$$

where we have factorized it into $2 \mathrm{D}$ components.

\subsection{H-momentum conservation}

In writing the above expression, we have applied the H-momentum conservation law, which has the following consequences. Firstly, since the H-momentum in each plane must be conserved, so must be their sum. But recall that the summed H-charge for each vertex operator is fixed, in particular, $V_{-1}$ has charge +1 , whereas $V_{-1 / 2}$ has charge $-1 / 2$. Subsequently, in the correlation function $\left\langle V_{-1} V_{-1 / 2} V_{-1 / 2} V_{0} \ldots V_{0}\right\rangle$ only the terms with zero summed Hmomentum in $V_{0}$ contribute. These are the $\bar{\partial} \bar{X}^{j}$ terms in (2.7). Therefore, we have $\mathcal{N}_{R}^{i}=0$ and $\sum_{i} \overline{\mathcal{N}}_{R}^{i}=\mathrm{L}-3[19,20,30]$.

\footnotetext{
${ }^{6}$ We thank Nana Cabo-Bizet and Damián Mayorga-Peña for raising this point and for discussions on it.
} 
Moreover, the H-momentum conservation in each plane reduces the correlation function to a form that factorizes over the three $2 \mathrm{D}$ directions, and determines how the right-moving oscillators are distributed amongst the three planes. In detail, although the correlation function is a sum of several terms due to the sums in the picture-changing operators of (2.7), all these terms are vanishing unless they satisfy the H-momentum conservation due to the result:

$$
\left\langle\prod_{\alpha=1}^{\mathrm{L}} e^{i q_{\alpha} \cdot H\left(\bar{z}_{\alpha}\right)}\right\rangle \sim \delta^{(5)}\left(\sum_{\alpha=1}^{\mathrm{L}} q_{\alpha}\right) \prod_{\alpha, \beta=1, \alpha<\beta}^{\mathrm{L}}\left(\bar{z}_{\alpha}-\bar{z}_{\beta}\right)^{\alpha^{\prime} q_{\alpha} \cdot q_{\beta} / 2} .
$$

This implies the condition

$$
\sum_{\alpha} q_{\mathrm{sh} \alpha}^{i}-\overline{\mathcal{N}}_{R}^{i}=1
$$

for $\overline{\mathcal{N}}_{R}^{i}$, which satisfy $\overline{\mathcal{N}}_{R}^{i} \geq 0$ and $\sum_{i} \overline{\mathcal{N}}_{R}^{i}=\mathrm{L}-3$. As we commented above, it turns out that in explicit models the above conditions can be satisfied for all couplings that satisfy both the point group selection rule and R-charge conservation. Indeed, it is easy to check that the couplings allowed by the point group selection rule, which violate H-momentum conservation (see e.g. [15] for a list of $\mathrm{H}$-momenta for the various twisted sectors in $\mathbb{Z}_{\mathrm{N}}$ orbifolds), also violate R-charge conservation. This is because the planes in which $\mathrm{H}$ momentum cannot be conserved turn out to be ones in which all fields are untwisted, with $q_{\mathrm{sh} \alpha}^{i}=0$. Moreover, in invariant planes massless modes do not have oscillators, so that $\mathrm{R}$-charge reduces to $\mathrm{H}$-momentum. Then it follows that $\sum_{\alpha=1}^{\mathrm{L}} \mathrm{R}_{\alpha}^{i}=0 \neq 1 \bmod \mathrm{N}^{i}$. We shall see this in an explicit model in section 4.3.

\subsection{Decomposition into classical and quantum parts}

To make further progress on the correlation function (3.1), we split the computation into classical and quantum parts. Indeed, the fields $X^{i}$ can be split into a classical instanton solution, which solves the equation of motion $\partial \bar{\partial} X_{\mathrm{cl}}^{i}=0$, and the quantum fluctuations about it:

$$
X^{i}(z, \bar{z})=X_{\mathrm{cl}}^{i}(z, \bar{z})+X_{\mathrm{qu}}^{i}(z, \bar{z}) .
$$

For a symmetric orbifold, which we will always assume, we have the relations:

$$
\begin{aligned}
& \bar{\partial} \bar{X}_{\mathrm{cl}}^{i}=\left(\partial X_{\mathrm{cl}}^{i}\right)^{*}, \\
& \bar{\partial} X_{\mathrm{cl}}^{i}=\left(\partial \bar{X}_{\mathrm{cl}}^{i}\right)^{*} .
\end{aligned}
$$

Of particular importance in what follows will be the explicit form for the classical solutions, which describe worldsheet instantons. The functional dependence of the classical solutions is determined by the local monodromy to be $[12,35,36]$

$$
\begin{aligned}
& \partial X_{\mathrm{cl}}^{i}(z)=\sum_{l=1}^{\mathrm{L}-\mathrm{M}^{i}-1} a_{l}^{i} h_{l}^{i}(z), \\
& \bar{\partial} X_{\mathrm{cl}}^{i}(\bar{z})=\sum_{l^{\prime}=1}^{\mathrm{M}^{i}-1} \bar{b}_{l^{\prime}}^{i}{\overline{h^{\prime}}}_{l^{\prime}}^{i}(\bar{z}),
\end{aligned}
$$


plus their complex conjugates (no summation over $i$ ). Here, the basis functions are

$$
\begin{aligned}
h_{l}^{i}(z) & =z^{l-1} \prod_{\alpha=1}^{\mathrm{L}}\left(z-z_{\alpha}\right)^{\mathbf{k}_{\alpha}^{i}-1}, & l & =1, \ldots, \mathrm{L}-\mathrm{M}^{i}-1, \\
{\overline{h^{\prime}}}_{l^{\prime}}^{\prime}(\bar{z}) & =\bar{z}^{l^{\prime}-1} \prod_{\alpha=1}^{\mathrm{L}}\left(\bar{z}-\bar{z}_{\alpha}\right)^{-\mathbf{k}_{\alpha}^{i},} & & l^{\prime}=1, \ldots, \mathrm{M}^{i}-1,
\end{aligned}
$$

and the coefficients $a_{l}^{i}, \bar{b}_{l^{\prime}}^{i}$ shall be computed below (cf. eq. (3.15)). Also, the integers $\mathrm{M}^{i}$ are given by $\mathrm{M}^{i}=\sum_{\alpha=1}^{\mathrm{L}} \mathbf{k}_{\alpha}^{i}$, and we have defined $\mathbf{k}_{\alpha}^{i}=k_{\alpha} v^{i} \bmod 1$, such that $0<\mathbf{k}_{\alpha}^{i} \leq 1$ in (3.6) and (3.8), and $0 \leq \mathbf{k}_{\alpha}^{i}<1$ in (3.7) and (3.9). The integers $\mathrm{M}^{i}$ give the range of $l, l^{\prime}$, and are determined from the requirement that the classical solutions correspond to a convergent classical action. ${ }^{7}$ Indeed, only the classical solutions with finite action contribute in the path integral, while the solutions leading to divergent action have no contribution. Then, the set of holomorphic basis functions (3.8) is non-empty when

$$
1+\sum_{\alpha}\left(-1+\mathbf{k}_{\alpha}^{i}\right)<0
$$

whereas the set of anti-holomorphic basis functions (3.9) is non-empty when

$$
1+\sum_{\alpha}\left(-\mathbf{k}_{\alpha}^{i}\right)<0
$$

Otherwise, holomorphic and/or anti-holomorphic worldsheet instantons are not relevant, and we must take instead the trivial solutions, respectively $\partial X_{\mathrm{cl}}^{i}=0$ and/or $\bar{\partial} X_{\mathrm{cl}}^{i}=0$.

For any coupling involving only twisted fields in the plane $i$ ( $\mathbf{k}_{\alpha}^{i}$ non-integers), the value of $\mathrm{M}^{i}$ lies between 1 and $\mathrm{L}-1$, and the total number of holomorphic plus anti-holomorphic functions is $\mathrm{L}-2$. The $\mathrm{L}-2$ coefficients, $a_{l}^{i}, \bar{b}_{l^{\prime}}^{i}$, which complete the description of the classical solutions are determined by the global monodromy conditions (the quantum part instead feels only the local monodromy):

$$
\begin{gathered}
\int_{\gamma_{p}} d z \partial X_{\mathrm{cl}}^{i}+\int_{\gamma_{p}} d \bar{z} \bar{\partial} X_{\mathrm{cl}}^{i}=\nu_{p}^{i}, \\
\int_{\gamma_{p}} d z \partial \bar{X}_{\mathrm{cl}}^{i}+\int_{\gamma_{p}} d \bar{z} \bar{\partial} \bar{X}_{\mathrm{cl}}^{i}=\bar{\nu}_{p}^{i},
\end{gathered}
$$

where $\gamma_{p}$ represent all possible net zero-twist closed loops enclosing the twist fields, and $\nu_{p}^{i}$ are elements of the corresponding cosets of the torus lattice. The number of independent equations arising from (3.12) is the number of independent net zero-twist closed loops, which was proven to be $\mathrm{L}-2$ in [36]. We can choose as a basis e.g. the loops used in $[13,14]$, where we encircle the fixed point $f_{p}$ clockwise $r_{p}$ times followed by the fixed point $f_{p+1}$ counterclockwise $s_{p}$ times. Here, $r_{p} k_{p}=s_{p} k_{p+1}$ mod N, with $r_{p}, s_{p}$ the smallest integers that satisfy this property, and $p=1, \ldots, \mathrm{L}-2$. The corresponding coset vectors, $\nu_{p}$, can then be written as

$$
\nu_{p}=\left(1-\theta^{r_{p} k_{p}}\right)\left(f_{p+1}-f_{p}+\lambda\right), \quad \lambda \in \Lambda .
$$

\footnotetext{
${ }^{7}$ Recall that the integral $\int d z d \bar{z} \prod_{\beta}\left|z-z_{\beta}\right|^{n_{\beta}}$ converges if and only if $n_{\beta}>-2$ for all $\beta$ and $\sum_{\beta} n_{\beta}<-2$.
} 
The global monodromy conditions thus stated are then just the required number to determine all the $\mathrm{L}-2$ coefficients, $a_{l}^{i}, \bar{b}_{l^{\prime}}^{i}$, which reduces to an exercise in linear algebra. Indeed, the solutions to (3.12) can be written in terms of the so-called period matrices, which have dimension $(\mathrm{L}-2) \times(\mathrm{L}-2)$ and components [36]

$$
\begin{array}{rlrl}
W_{p}^{i l} & =\int_{\gamma_{p}} d z h_{l}^{i}(z), & l & l, \ldots, \mathrm{L}-\mathrm{M}^{i}-1, \\
W_{p}^{i\left(\mathrm{~L}-\mathrm{M}^{i}-1+l^{\prime}\right)} & =\int_{\gamma_{p}} d \bar{z}{\overline{h^{\prime}}}_{l^{\prime}}^{i}(\bar{z}), & & l^{\prime}=1, \ldots, \mathrm{M}^{i}-1 .
\end{array}
$$

In terms of these matrices, the coefficients are

$$
\begin{aligned}
a_{l}^{i} & =\nu_{p}^{i}\left(W^{-1}\right)_{l}^{i p}, \\
\bar{b}_{l^{\prime}}^{i} & =\bar{\nu}_{p}^{i}\left(W^{-1}\right)_{L-M^{i}-1+l^{\prime}}^{i p} .
\end{aligned}
$$

Thus for a particular classical instanton solution, the coefficients $a_{l}^{i}, b_{l^{\prime}}^{i}$ are particular linear combinations of the coset vectors $\left\{\nu_{p}^{i}\right\}$.

One more comment on the global monodromy conditions is in order here. In general, there is also an additional consistency condition [14] arising from the space group selection rule and the $(\mathrm{L}-1)$ th $\left(r_{p}, s_{p}\right)$-loop. Indeed, the $(\mathrm{L}-1)$ th loop is not linearly independent because the sum of all the $(\mathrm{L}-1)$ loops can be pulled around the worldsheet sphere and shrunk to zero, giving rise to the space group selection rule. However, this extra consistency condition can restrict further the coset vectors appearing in (3.13), (3.15). ${ }^{8}$ For instance, for the 3-point couplings it turns out that the coset vectors are restricted to [14]:

$$
\nu=\left(1-\theta^{r_{1} k_{1}}\right)\left(f_{2}-f_{1}-\tau_{2}+\tau_{1}+\left(1-\theta^{k_{1}+k_{2}}\right)\left(1-\theta^{\operatorname{gcd}\left(k_{1}, k_{2}\right)}\right)^{-1} \lambda\right), \quad \lambda \in \Lambda,
$$

where $\tau_{1,2}$ are the torus lattice vectors that appear in the space group selection rule (2.17) and gcd stands for greatest common denominator.

Having split the fields into their classical and quantum parts, the correlation function $\mathcal{F}=\prod_{i=1}^{3} \mathcal{F}^{i}$ also splits as

$$
\begin{aligned}
\mathcal{F}^{i}=\sum_{r=0}^{\mathcal{N}_{L}^{i}} & \left(\begin{array}{c}
\mathcal{N}_{L}^{i} \\
r
\end{array}\right) \sum_{s=0}^{\overline{\mathcal{N}}_{L}^{i}}\left(\begin{array}{c}
\overline{\mathcal{N}}_{L}^{i} \\
s
\end{array}\right) \sum_{t=0}^{\overline{\mathcal{N}}_{R}^{i}}\left(\begin{array}{c}
\overline{\mathcal{N}}_{R}^{i} \\
t
\end{array}\right) \sum_{X_{\mathrm{cl}}^{i}} e^{-S_{\mathrm{cl}}^{i}} \\
& \times\left\langle\left(\partial X_{\mathrm{cl}}^{i}\right)^{\mathcal{N}_{L}^{i}-r}\left(\partial X_{\mathrm{qu}}^{i}\right)^{r}\left(\partial \bar{X}_{\mathrm{cl}}^{i}\right)^{\overline{\mathcal{N}}_{L}^{i}-s}\left(\partial \bar{X}_{\mathrm{qu}}^{i}\right)^{s}\left(\bar{\partial} \bar{X}_{\mathrm{cl}}^{i}\right)^{\overline{\mathcal{N}}_{R}^{i}-t}\left(\bar{\partial} \bar{X}_{\mathrm{qu}}^{i}\right)^{t}\right\rangle_{\sigma_{\left(k_{1}, f_{1}\right.}^{i} \cdots \sigma_{\left(k_{\mathrm{L}}, f_{\mathrm{L}}\right)}^{i}},
\end{aligned}
$$

where $\left(\mathcal{N}_{L}^{i}\right)$ and so on are the binomial coefficients, the classical action is given by

$$
S_{\mathrm{cl}}^{i}=\frac{1}{8 \pi} \int d^{2} z\left(\left|\partial X_{\mathrm{cl}}^{i}\right|^{2}+\left|\partial \bar{X}_{\mathrm{cl}}^{i}\right|^{2}\right)
$$

and we have defined

$$
\begin{aligned}
\left\langle\left(\partial X_{\mathrm{cl}}^{i}\right)^{\mathcal{N}_{L}^{i}-r}\left(\partial X_{\mathrm{qu}}^{i}\right)^{r}\left(\partial \bar{X}_{\mathrm{cl}}^{i}\right)^{\overline{\mathcal{N}}_{L}^{i}-s}\left(\partial \bar{X}_{\mathrm{qu}}^{i}\right)^{s}\left(\bar{\partial} \bar{X}_{\mathrm{cl}}^{i}\right)^{\overline{\mathcal{N}}_{R}^{i}-t}\left(\bar{\partial} \bar{X}_{\mathrm{qu}}^{i}\right)^{t}\right\rangle_{\sigma_{\left(k_{1}, f_{1}\right.}^{i} \cdots \sigma_{\left(k_{\mathrm{L}}, f_{\mathrm{L}}\right)}} \\
=\left(\partial X_{\mathrm{cl}}^{i}\right)^{\mathcal{N}_{L}^{i}-r}\left(\partial \bar{X}_{\mathrm{cl}}^{i}\right)^{\mathcal{N}_{L}^{i}-s}\left(\bar{\partial} \bar{X}_{\mathrm{cl}}^{i}\right)^{\mathcal{N}_{R}^{i}-t} \\
\quad \times \int \mathcal{D} X_{\mathrm{qu}}^{i} e^{-S_{\mathrm{qu}}^{i}}\left(\partial X_{\mathrm{qu}}^{i}\right)^{r}\left(\partial \bar{X}_{\mathrm{qu}}^{i}\right)^{s}\left(\bar{\partial} \bar{X}_{\mathrm{qu}}^{i}\right)^{t} \sigma_{\left(k_{1}, f_{1}\right)}^{i} \cdots \sigma_{\left(k_{\mathrm{L}}, f_{\mathrm{L}}\right)}^{i}
\end{aligned}
$$

\footnotetext{
${ }^{8}$ This condition has not been considered in previous works on higher order couplings [18, 37].
} 
We will see that it is not necessary to compute this expression explicitly in order to derive the selection rules. Indeed, we now use the general form of the correlation function (3.17) to deduce that many couplings are forced to be vanishing.

\section{$3.3 \quad 3$-point couplings}

Let us begin by considering general 3-point couplings. In this case we do not have to change pictures, since we have simply the coupling $\left\langle V_{-1 / 2} V_{-1 / 2} V_{-1}\right\rangle$. Thus, there are no right-moving oscillators, $\overline{\mathcal{N}}_{R}^{i}=0$. At the same time, if some of the participating fields have excited string modes, we do have left-moving oscillators. In this case, there are extra selection rules.

\subsubsection{A new rule: "Rule 5"}

Referring to eqs. (3.6) and (3.7), for 3-point couplings there exist either non-trivial holomorphic classical solutions $\left(\sum_{\alpha} \mathbf{k}_{\alpha}^{i}<2\right.$ where $\left.0<\mathbf{k}_{\alpha}^{i} \leq 1\right)$ or non-trivial anti-holomorphic solutions $\left(\sum_{\alpha} \mathbf{k}_{\alpha}^{i}>1\right.$ where $\left.0 \leq \mathbf{k}_{\alpha}^{i}<1\right)$ or neither, but not both. Consider the $i$-th plane and suppose e.g. that only the holomorphic classical solutions are non-trivial. Then all the terms in the sum over $s$ in (3.17) vanish apart from the one with $s=\overline{\mathcal{N}}_{L}^{i}$. Next take the sum over $r$. All these terms are zero apart from the one with $r=s$, which can be straightforwardly deduced from the basic OPE [31]

$$
X^{M}(z, \bar{z}) X^{N}(w, \bar{w}) \sim-\eta^{M N} \ln |z-w|^{2}, \quad M, N=1, \ldots, 10 .
$$

Thus we require $\mathcal{N}_{L}^{i} \geq \overline{\mathcal{N}}_{L}^{i}$ for the coupling to be non-vanishing, which provides a new selection rule. Correlation functions that survive this Rule 5 reduce to:

$$
\mathcal{F}^{i}=\left(\begin{array}{c}
\mathcal{N}_{L}^{i} \\
\overline{\mathcal{N}}_{L}^{i}
\end{array}\right) \sum_{X_{\mathrm{cl}}^{i}} e^{-S_{\mathrm{cl}}^{i}}\left(\partial X_{\mathrm{cl}}^{i}\right)^{\mathcal{N}_{L}^{i}-\overline{\mathcal{N}}_{L}^{i}} \int \mathcal{D} X_{\mathrm{qu}}^{i} e^{-S_{\mathrm{qu}}^{i}}\left(\partial X_{\mathrm{qu}}^{i}\right)^{\overline{\mathcal{N}}_{L}^{i}}\left(\partial \bar{X}_{\mathrm{qu}}^{i}\right)^{\overline{\mathcal{N}}_{L}^{i}} \sigma_{\left(k_{1}, f_{1}\right)}^{i} \sigma_{\left(k_{2}, f_{2}\right)}^{i} \sigma_{\left(k_{3}, f_{3}\right)}^{i}
$$

Following the same steps for the case that only anti-holomorphic instantons are allowed, it is easy to see that the correlation functions vanish unless $\overline{\mathcal{N}}_{L}^{i} \geq \mathcal{N}_{L}^{i}$. Finally, if neither holomorphic nor anti-holomorphic instanton solutions exist, then the correlation functions vanish unless $\mathcal{N}_{L}^{i}=\overline{\mathcal{N}}_{L}^{i}$.

\subsubsection{Twist invariance}

Assume now for concreteness a coupling for which only holomorphic instantons are allowed, and take the case $\mathcal{N}_{L}^{i}>\overline{\mathcal{N}}_{L}^{i}$. The correlation function reduces as above to eq. (3.21), which we rewrite in the shorthand:

$$
\mathcal{F}^{i}=\sum_{X_{\mathrm{cl}}^{i}} e^{-S_{\mathrm{cl}}^{i}}\left(\partial X_{\mathrm{cl}}^{i}\right)^{\mathcal{N}_{L}^{i}-\overline{\mathcal{N}}_{L}^{i}} \cdot \mathcal{Z}_{\mathrm{qu}}^{i}
$$

Now, recall the explicit form of the holomorphic instantons for the 3-point couplings:

$$
\partial X_{\mathrm{cl}}^{i}=a^{i} h^{i}(z) .
$$


The coefficient $a^{i}=\tilde{a}^{i} \nu^{i}$ is proportional to vectors that are given in eq. (3.16). This set of vectors enjoys the $\mathbb{Z}_{\mathrm{N}^{i}}$ twist symmetry of the $2 \mathrm{D}$ plane, and can thus be arranged into sets of $\mathrm{N}^{i}$ vectors with equal length as follows: $\nu^{i}=\left\{r, r \omega, r \omega^{2}, \ldots, r \omega^{\mathrm{N}^{i}-1}\right\}$, $\left\{2 r, 2 r \omega, 2 r \omega^{2}, \ldots, 2 r \omega^{\mathrm{N}^{i}-1}\right\}, \ldots$, with $\omega=e^{2 \pi i / \mathrm{N}^{i}}$. Contributions to the sum over instantons in (3.22) can be similarly arranged:

$$
\begin{aligned}
\mathcal{F}^{i}= & e^{-\frac{r^{2}\left|\tilde{a}^{i}\right|^{2}}{8 \pi} \int\left|h^{i}(z)\right|^{2}}\left(h^{i}\right)^{\mathcal{N}_{L}^{i}-\overline{\mathcal{N}}_{L}^{i}}\left(r \tilde{a}^{i}\right)^{\mathcal{N}_{L}^{i}-\overline{\mathcal{N}}_{L}^{i}} \\
& \times\left(1^{\mathcal{N}_{L}^{i}-\overline{\mathcal{N}}_{L}^{i}}+\omega^{\mathcal{N}_{L}^{i}-\overline{\mathcal{N}}_{L}^{i}}+\cdots+\omega^{\left(\mathrm{N}^{i}-1\right)\left(\mathcal{N}_{L}^{i}-\overline{\mathcal{N}}_{L}^{i}\right)}\right) \cdot \mathcal{Z}_{\mathrm{qu}}^{i}+\cdots
\end{aligned}
$$

Using the geometric series we see that the correlation function vanishes unless

$$
\mathcal{N}_{L}^{i}-\overline{\mathcal{N}}_{L}^{i}=0 \bmod \mathrm{N}^{i}
$$

This is twist invariance.

\subsubsection{Remembering Rule 4}

Continuing, for a given instanton solution (3.23), when all fields are localized at the same fixed point in the $i$-th plane, the coefficient $a^{i}=\tilde{a}^{i} \nu^{i}$ is actually proportional to vectors belonging to (a sublattice of) the original torus lattice, which can be read from (3.16). Suppose the corresponding sublattice has a $\mathbb{Z}_{\mathrm{K}}$ automorphism group. Just as before, the lattice vectors can be arranged into sets of $\mathrm{K}$ vectors with equal length as follows: $\nu^{i}=\left\{r, r \omega, r \omega^{2}, \ldots, r \omega^{\mathrm{K}-1}\right\},\left\{2 r, 2 r \omega, 2 r \omega^{2}, \ldots, 2 r \omega^{\mathrm{K}-1}\right\}, \ldots$, now with $\omega=e^{2 \pi i / \mathrm{K}}$. Contributions to the sum over instantons in (3.22) are arranged as before:

$$
\begin{aligned}
\mathcal{F}^{i}= & e^{-\frac{r^{2}\left|\tilde{a}^{i}\right|^{2}}{8 \pi} \int\left|h^{i}(z)\right|^{2}}\left(h^{i}\right)^{\mathcal{N}_{L}^{i}-\overline{\mathcal{N}}_{L}^{i}}\left(r \tilde{a}^{i}\right)^{\mathcal{N}_{L}^{i}-\overline{\mathcal{N}}_{L}^{i}} \\
& \times\left(1^{\mathcal{N}_{L}^{i}-\overline{\mathcal{N}}_{L}^{i}}+\omega^{\mathcal{N}_{L}^{i}-\overline{\mathcal{N}}_{L}^{i}}+\cdots+\omega^{(\mathrm{K}-1)\left(\mathcal{N}_{L}^{i}-\overline{\mathcal{N}}_{L}^{i}\right)}\right) \cdot \mathcal{Z}_{\mathrm{qu}}^{i}+\cdots .
\end{aligned}
$$

Now, let us assume a $\mathbb{Z}_{3}$ twist in the $i$-th plane. Twist invariance or R-charge conservation already requires that $\mathcal{N}_{L}^{i}-\overline{\mathcal{N}}_{L}^{i}=0 \bmod 3$ for a non-vanishing coupling. The $\mathbb{Z}_{3}$ planes, however, are constructed on a lattice with $\mathbb{Z}_{6}$ symmetry, namely $\mathrm{SU}(3)$ or $G_{2}$. As above, it is easy to work out from (3.25) that correlation functions are actually then vanishing unless $\mathcal{N}_{L}^{i}-\overline{\mathcal{N}}_{L}^{i}=0 \bmod 6$. Analogous statements can of course be made when it is the anti-holomorphic instantons that are allowed. Similar arguments could also apply to a $\mathbb{Z}_{2}$ twist, on a lattice plane with automorphism group $\mathbb{Z}_{4}$ or $\mathbb{Z}_{6}$. However, it happens that in explicit models, planes with a $\mathbb{Z}_{2}$ twist are invariant planes for 3-point couplings, i.e. at least one field in the coupling is untwisted on this plane. It then follows that no instanton solutions are allowed there, and Rule 5 imposes the stronger condition, $\mathcal{N}_{L}^{i}-\overline{\mathcal{N}}_{L}^{i}=0$.

In summary, we have an extra selection rule whenever the symmetry group of the lattice governing the couplings is larger than the point group. This rule was first introduced in the literature in $[11,19]$, and discussed in $[20,38]$. Before it came to be forgotten it was known as Rule 4. We can see crystallographically that it is relevant for the following types of orbifold planes: a $\mathbb{Z}_{2}$ twist on an $\mathrm{SO}(4)$ or $\mathrm{SO}(5)$ torus lattice; a $\mathbb{Z}_{2}$ twist on an $\mathrm{SU}(3)$ or $G_{2}$ torus lattice; a $\mathbb{Z}_{3}$ twist on an $\mathrm{SU}(3)$ or $G_{2}$ torus lattice. $^{9}$

\footnotetext{
${ }^{9}$ Note that the sublattices appearing in the various instanton solutions turn out to have the same automorphism symmetry as the original torus lattice, at least for the planes with $\mathbb{Z}_{2}$ and $\mathbb{Z}_{3}$ twists.
} 


\subsection{Higher order couplings}

We are now ready to discuss general L-point couplings, L $>3$. For these higher order couplings we necessarily have L-3 right-moving oscillators, distributed amongst the three orbifold planes, from the picture-changing operation. However, it follows from the OPE (3.20) that all terms in the sum over $t$ in eq. (3.17) vanish apart from $t=0$. The discussion of the selection rules is then a straightforward generalization of that for the 3-point couplings.

\subsubsection{Rule 5}

Let us first state Rule 5 . There are several different cases.

Consider the $i$-th plane. If neither the holomorphic nor anti-holomorphic solutions can be non-trivial, then we require $\overline{\mathcal{N}}_{R}^{i}=0$ and $\mathcal{N}_{L}^{i}=\overline{\mathcal{N}}_{L}^{i}$ and the correlation function takes the form:

$$
\mathcal{F}^{i}=\int \mathcal{D} X_{\mathrm{qu}}^{i} e^{-S_{\mathrm{qu}}^{i}}\left(\partial X_{\mathrm{qu}}^{i}\right)^{\mathcal{N}_{L}^{i}}\left(\partial \bar{X}_{\mathrm{qu}}^{i}\right)^{\mathcal{N}_{L}^{i}} \sigma_{\left(k_{1}, f_{1}\right)}^{i} \ldots \sigma_{\left(k_{\mathrm{L}}, f_{\mathrm{L}}\right)}^{i} .
$$

If holomorphic instantons are allowed, but anti-holomorphic instantons are not, then $\mathcal{N}_{L}^{i} \geq$ $\overline{\mathcal{N}}_{L}^{i}$, and the coupling is given by:

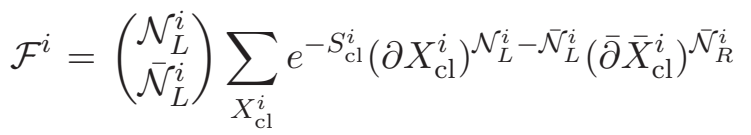

$$
\begin{aligned}
& \int \mathcal{D} X_{\mathrm{qu}}^{i} e^{-S_{\mathrm{qu}}^{i}}\left(\partial X_{\mathrm{qu}}^{i}\right)^{\overline{\mathcal{N}}_{L}^{i}}\left(\partial \bar{X}_{\mathrm{qu}}^{i}\right)^{\overline{\mathcal{N}}_{L}^{i}} \sigma_{\left(k_{1}, f_{1}\right)}^{i} \ldots \sigma_{\left(k_{\mathrm{L}}, f_{\mathrm{L}}\right)}^{i} .
\end{aligned}
$$

If instead only anti-holomorphic instantons are allowed, then $\overline{\mathcal{N}}_{R}^{i}=0$ and $\mathcal{N}_{L}^{i} \leq \overline{\mathcal{N}}_{L}^{i}$, and

$$
\begin{aligned}
\mathcal{F}^{i}=\left(\begin{array}{c}
\overline{\mathcal{N}}_{L}^{i} \\
\mathcal{N}_{L}^{i}
\end{array}\right) & \sum_{X_{\mathrm{cl}}^{i}} e^{-S_{\mathrm{cl}}^{i}\left(\partial \bar{X}_{\mathrm{cl}}^{i}\right)^{\mathcal{\mathcal { N }}_{L}^{i}-\mathcal{N}_{L}^{i}}} \\
& \int \mathcal{D} X_{\mathrm{qu}}^{i} e^{-S_{\mathrm{qu}}^{i}}\left(\partial X_{\mathrm{qu}}^{i}\right)^{\mathcal{N}_{L}^{i}}\left(\partial \bar{X}_{\mathrm{qu}}^{i}\right)^{\mathcal{N}_{L}^{i}} \sigma_{\left(k_{1}, f_{1}\right)}^{i} \ldots \sigma_{\left(k_{\mathrm{L}}, f_{\mathrm{L}}\right)}^{i} .
\end{aligned}
$$

If both holomorphic and anti-holomorphic instantons are allowed, then all the couplings survive Rule 5, and their correlation functions take the form:

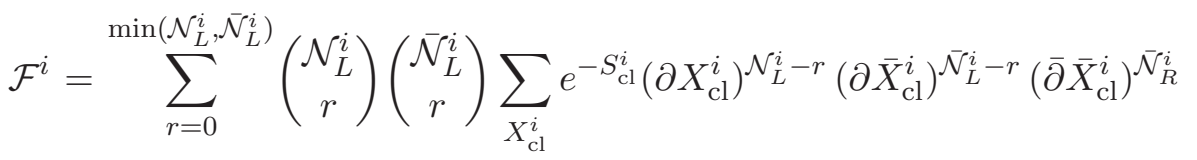

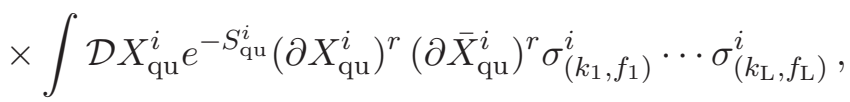

where min stands for the smallest number.

Although the above rule is applicable to the couplings including both the twisted and untwisted sectors, we need a remark for the couplings that include only the fully untwisted fields. If all fields are untwisted, there are no instantonic solutions on any plane. Then, Rule 5 requires $\overline{\mathcal{N}}_{R}^{i}=0$ for all of $i=1,2,3$. This implies

$$
\mathrm{L}-3=\sum_{i} \overline{\mathcal{N}}_{R}^{i}=0 .
$$


That is, only the 3-point couplings are allowed among untwisted fields, whilst higher order couplings are forbidden $[39,40]$. In particular, the $U_{1} U_{2} U_{3}$ couplings are allowed by the $\mathrm{H}$-momentum conservation, where we recall that $U_{1}, U_{2}$, and $U_{3}$ denote the untwisted sectors with the H-momenta, $q_{\mathrm{sh}}^{i}=(1,0,0),(0,1,0)$, and $(0,0,1)$, respectively. Note that untwisted fields are 10D bulk modes. Dimensional reduction from 10D supersymmetric Yang-Mills theory leads to the same result, that is, only the $U_{1} U_{2} U_{3}$ couplings are allowed among untwisted matter couplings, but higher order couplings are forbidden by $4 \mathrm{D} \mathcal{N}=4$ supersymmetry. See however section 3.5 for a way to evade this restriction.

\subsubsection{Rule 4}

Twist invariance generalised to the L-point couplings gives rise to the condition (2.13). We must now apply Rule 4 to all the couplings that have survived so far. Recall the explicit form for the instanton solutions:

$$
\begin{aligned}
& \partial X_{\mathrm{cl}}^{i}(z)=\sum_{l=1}^{\mathrm{L}-\mathrm{M}^{i}-1} a_{l}^{i} h_{l}^{i}(z)=\sum_{p=1}^{\mathrm{L}-2} \sum_{l=1}^{\mathrm{L}-\mathrm{M}^{i}-1} \nu_{p}^{i}\left(W^{-1}\right)_{l}^{i p} h_{l}^{i}(z)=\sum_{p=1}^{\mathrm{L}-2} \nu_{p}^{i} h_{p}^{i}(z), \\
& \bar{\partial} X_{\mathrm{cl}}^{i}(\bar{z})=\sum_{l^{\prime}=1}^{\mathrm{M}^{i}-1} b_{l^{\prime}}^{i} h_{l^{\prime}}^{i}(\bar{z})=\sum_{p=1}^{\mathrm{L}-2} \sum_{l^{\prime}=1}^{\mathrm{M}^{i}-1} \nu_{p}^{i}\left(W^{-1}\right)_{\mathrm{L}-\mathrm{M}^{i}-1+l^{\prime}}^{i p} h_{l^{\prime}}^{i}(\bar{z})=\sum_{p=1}^{\mathrm{L}-2} \nu_{p}^{i} h_{p}^{\prime i}(\bar{z}),
\end{aligned}
$$

plus their complex conjugates. Here, the vectors $\nu_{p}^{i}$ belong to particular cosets of the $i$ th torus lattice and $h_{p}^{i}, h_{p}^{\prime i}$ are defined in the obvious way via the above equations. Thus, the instanton solutions mediating higher order couplings between twisted fields at the same fixed point are linear combinations of several torus sublattice vectors. In principle, different sublattices might each have different automorphism groups, but for the $\mathbb{Z}_{2}$ twist and $\mathbb{Z}_{3}$ twist planes of interest to us, all sublattices have the same symmetry as the original torus lattice.

Let us then state the consequences of these lattice symmetries. For a plane with $\mathbb{Z}_{3}$ twist on a $\mathbb{Z}_{6}$ lattice, the extra $\mathbb{Z}_{2}$ symmetry implies that the sublattice vectors can be paired up as $\left(\nu_{p}^{i},-\nu_{p}^{i}\right)$. Put together with the twist invariance condition $\mathcal{N}_{L}^{i}-\overline{\mathcal{N}}_{L}^{i}-$ $\overline{\mathcal{N}}_{R}^{i}=0 \bmod 3$, this pairing implies that the sums in (3.17) vanish unless moreover ${ }^{10}$ $\mathcal{N}_{L}^{i}-\overline{\mathcal{N}}_{L}^{i}-\overline{\mathcal{N}}_{R}^{i}=0 \bmod 6$. For a plane with $\mathbb{Z}_{2}$ twist on a $\mathbb{Z}_{4}$ lattice, the lattice and twist symmetries together imply that $\mathcal{N}_{L}^{i}-\overline{\mathcal{N}}_{L}^{i}-\overline{\mathcal{N}}_{R}^{i}=0 \bmod 4$ for non-trivial couplings. Finally, for a $\mathbb{Z}_{2}$ twist plane on a $\mathbb{Z}_{6}$ lattice, non-vanishing correlation functions again meet the condition $\mathcal{N}_{L}^{i}-\overline{\mathcal{N}}_{L}^{i}-\overline{\mathcal{N}}_{R}^{i}=0 \bmod 6$. Notice thus that for orbifolds with a $\mathbb{Z}_{2}$ plane, and hence a complex structure, the oscillator couplings depend on the complex structure modulus via the sum over lattice vectors, and for special values of the complex structure modulus an enhanced lattice symmetry forces additional couplings to vanish.

\footnotetext{
${ }^{10}$ In detail, the sum over classical solutions in (3.17) translates into $\mathrm{L}-2$ sums over the sublattice vectors $\left\{\nu_{p}^{i}\right\}$. The terms $\nu_{p}^{i}$ in the sums cancel with the terms $-\nu_{p}^{i}$ unless $\mathcal{N}_{L}^{i}-r+\overline{\mathcal{N}}_{L}^{i}-r-\overline{\mathcal{N}}_{R}^{i}=0 \bmod 2$. Meanwhile, the point group symmetry implies that couplings vanish unless $\mathcal{N}_{L}^{i}-\overline{\mathcal{N}}_{L}^{i}-\overline{\mathcal{N}}_{R}^{i}=0 \bmod 3$. These two conditions together imply $\mathcal{N}_{L}^{i}-\overline{\mathcal{N}}_{L}^{i}-\overline{\mathcal{N}}_{R}^{i}=0 \bmod 6$. Similar arguments work also for the other cases.
} 
Couplings containing untwisted fields in the plane are also sensitive to Rule 4, unless all fields are untwisted. This follows from the fact that only the twisted fields comprised in a coupling contribute non-trivially to the instanton solutions in (3.31), (3.32), which inherit the lattice symmetry. If all fields are untwisted in the plane, there are no instantonic solutions there and no additional symmetries emerge. If all fields are fully untwisted, higher order couplings are forbidden by Rule 5. Finally, note that, in the case that twisted fields are localized at different fixed points, the symmetry of the coefficients of the instantonic solutions is only the one of the twist, so Rule 4 has no effect.

\subsection{On effective couplings}

Our interest is in how to identify possible non-vanishing terms, $\Phi^{\mathrm{L}}$, in the holomorphic matter superpotential, $W$, of the low energy effective field theory. We do so by studying the tree-level correlation functions between holomorphic matter fields of the form $\left\langle V_{F} V_{F} V_{B}^{\mathrm{L}-2}\right\rangle$, where $V_{F}$ and $V_{B}$ stand for the corresponding fermionic and bosonic vertex operators.

If a coupling is allowed by our selection rules, it may still be vanishing for other reasons. Conversely if a given superpotential matter coupling $\left\langle\psi_{1} \psi_{2} \phi_{3} \ldots \phi_{\mathrm{L}}\right\rangle$ is forbidden by the selection rules, it may be possible to generate it below some energy scale via an allowed coupling $\left\langle\psi_{1} \psi_{2} \phi_{3} \ldots \phi_{\mathrm{L}} s\right\rangle$, for $s$ some singlet fields, if the singlet fields acquire vevs at that scale.

Another way to generate effective couplings is via holomorphic terms in the Kähler potential. For example, in the presence of a $\mathbb{Z}_{2}$ plane with Kähler modulus $T^{3}$, complex structure modulus $Z$, and two complex Wilson line moduli, $M_{1}$ and $M_{2}$, the Kähler potential takes the form $[43-45]:^{11}$

$$
K=-\log \left(\left(T^{3}+\bar{T}^{3}\right)(Z+\bar{Z})-\frac{1}{2}\left(M_{1}+\bar{M}_{2}\right)\left(\bar{M}_{1}+M_{2}\right)\right)
$$

Expanding the logarithm, we see that the term $e^{K / 2} \partial_{\alpha} \partial_{\beta} W \psi^{\alpha} \psi^{\beta}$ in the supergravity Lagrangian, corresponds then not only to a coupling $\psi_{1} \psi_{2} \phi_{3} \ldots \phi_{\mathrm{L}}$ from the superpotential, but also to a coupling $\psi_{1} \psi_{2} \phi_{3} \ldots \phi_{\mathrm{L}} \phi_{M_{1}} \phi_{M_{2}}$ (though not to the scattering amplitude $\left.\left\langle V_{\psi_{1}} V_{\psi_{2}} V_{\phi_{3}} \ldots V_{\phi_{\mathrm{L}}} V_{\phi_{M_{1}}} V_{\phi_{M_{2}}}\right\rangle\right)$. Note that the latter coupling could be interpreted as an effective superpotential term, once the moduli fields have been integrated out.

It is interesting to note that effective superpotential couplings descending from the Kähler potential after moduli stabilization are expected to satisfy R-charge conservation, since this corresponds to Lorentz symmetries that survive the orbifold compactification. In contrast, Rules 4 and 5 emerge from the structure of the tree-level string correlation functions between holomorphic matter fields $\left\langle V_{F} V_{F} V_{B}^{\mathrm{L}-2}\right\rangle$, which we use to identify the superpotential. Therefore, the effective couplings obtained after integrating out the moduli may violate Rules 4 and 5, as in the example above. In order to identify which of these effective couplings are non-vanishing, one must combine the allowed superpotential couplings and the allowed contributions to the Kähler potential.

\footnotetext{
${ }^{11}$ For phenomenological applications of this Kähler potential in orbifolds see e.g. [46, 47].
} 


\section{A concrete example: the $T^{6} / \mathbb{Z}_{6-I I}$ orbifold}

In this section, we study the L-point couplings in an explicit model, and in particular the role of Selection Rules 4 and 5 . We choose the $T^{6} / \mathbb{Z}_{6-I I}$ orbifold with twist vector $v^{i}=\frac{1}{6}(1,2,-3)$ and underlying torus lattice $G_{2} \times \mathrm{SU}(3) \times \mathrm{SO}(4)$. This orbifold has received much attention in the recent literature, since for certain gauge embeddings and Wilson lines it can give rise to a massless spectrum containing the MSSM and no chiral exotics. The first, second and third planes have respectively $\mathbb{Z}_{6}, \mathbb{Z}_{3}$ and $\mathbb{Z}_{2}$ twist symmetries, but $\mathbb{Z}_{6}$, $\mathbb{Z}_{6}$ and $\mathbb{Z}_{4}$ lattice symmetries.

In principle, since $T^{6} / \mathbb{Z}_{6-I I}$ is a non-prime orbifold, we must take care to construct the physical twist-invariant states by taking linear combinations of the basic twisted states. However, the calculation for the corresponding correlation functions reduces in any case to computing auxiliary correlation functions of the type discussed above. Moreover, the auxiliary correlations all involve the same sets of quantum numbers, apart from the localization of twisted states in the first plane. Therefore, if Rules 4 and 5 eliminate one term in the linear combination of auxiliary correlation functions, they eliminate every term.

Below, we will use the H-momentum of the $\theta, \theta^{2}, \theta^{3}, \theta^{4}$ twisted-sector states, which are, respectively, $q_{\mathrm{sh} \alpha}^{i}=\left(\frac{1}{6}, \frac{1}{3}, \frac{1}{2}\right),\left(\frac{1}{3}, \frac{2}{3}, 0\right),\left(\frac{1}{2}, 0, \frac{1}{2}\right),\left(\frac{2}{3}, \frac{1}{3}, 0\right)$ (the $\theta^{5}$ sector does not contain positive chiral states).

\subsection{3-point couplings}

We begin with the 3 -point couplings, $\theta^{k_{1}} \theta^{k_{2}} \theta^{k_{3}}$. After applying the standard selection rules, the surviving couplings are of kind $\theta \theta \theta^{4}$ and $\theta \theta^{2} \theta^{3}$. Using the explicit H-momenta for the twisted states given above, it is easy to see that the H-momentum is automatically conserved for these couplings. Similarly, we can show that the H-momentum is automatically conserved for all of the 3-point couplings allowed by the standard selection rule in (factorizable) $T^{6} / \mathbb{Z}_{3}, T^{6} / \mathbb{Z}_{4}$, and $T^{6} / \mathbb{Z}_{6-I}$ orbifolds.

Consider now the properties of the classical solutions. Following the discussion around (3.6)-(3.9), for the $\theta \theta \theta^{4}$ coupling, the allowed instanton solutions are the holomorphic ones in the first and second planes. The fact that in the third plane there is no non-trivial classical solution meets with our intuition, since the third plane is untwisted for the $\theta \theta \theta^{4}$ coupling, implying that there is no worldsheet instanton contribution to the coupling. Similarly, for the $\theta \theta^{2} \theta^{3}$ coupling only the holomorphic instantons in the first plane are allowed. Since several of the classical solutions are forced to be vanishing, Rule 5 eliminates various couplings.

Moreover, for the $\theta \theta \theta^{4}$ coupling, when we compute explicitly the coefficients $a^{1}, a^{2}$ from the global monodromy conditions, we find that the sets of classical solutions have an extra $\mathbb{Z}_{2}$ symmetry in the second plane, descending from that of the $\mathrm{SU}(3)$ torus lattice. Therefore Rule 4 can forbid some of the couplings that would be mediated by allowed worldsheet instantons in the second plane.

We can now write down the selection rules. In the cases where there are no oscillator states involved, only the standard selection rules are relevant. In the presence of oscillators, we have in addition 
- For $\theta \theta \theta^{4}$ : couplings are non-vanishing only if the following three conditions are satisfied (i) $\mathcal{N}_{L}^{1} \geq \overline{\mathcal{N}}_{L}^{1}$ (ii) $\mathcal{N}_{L}^{2} \geq \overline{\mathcal{N}}_{L}^{2}$ (iii) $\overline{\mathcal{N}}_{L}^{3}=\mathcal{N}_{L}^{3}$. Moreover, when all fields are at the same fixed point in the second plane, Rule 4 imposes $\mathcal{N}_{L}^{2}-\overline{\mathcal{N}}_{L}^{2}=0 \bmod 6$.

- For $\theta \theta^{2} \theta^{3}$ : couplings are non-vanishing only if (i) $\mathcal{N}_{L}^{1} \geq \overline{\mathcal{N}}_{L}^{1}$ (ii) $\mathcal{N}_{L}^{2}=\overline{\mathcal{N}}_{L}^{2}$ (iii) $\mathcal{N}_{L}^{3}=\overline{\mathcal{N}}_{L}^{3}$.

\subsection{4-point couplings}

Before considering the general case, we discuss in detail the 4-point couplings, $\theta^{\mathbf{k}_{1}} \theta^{\mathbf{k}_{2}} \theta^{\mathbf{k}_{3}} \theta^{\mathbf{k}_{4}}$. There are eleven types of couplings between four twisted fields, but upon application of the standard selection rules it turns out that those of interest are $\theta^{4} \theta^{4} \theta^{3} \theta, \theta^{3} \theta \theta \theta$ and $\theta^{2} \theta^{2} \theta \theta$. Again, it is easy to check that H-momentum is conserved for these couplings.

As before, we begin by studying the properties of the classical solutions. For $\theta^{4} \theta^{4} \theta^{3} \theta$, the allowed instantons turn out to be (i) holomorphic and anti-holomorphic instantons in the first plane (ii) holomorphic instantons in the second plane. For $\theta^{3} \theta \theta \theta$, the allowed instantons are: (i) holomorphic instantons in the first plane (ii) holomorphic instantons in the second plane (iii) holomorphic and anti-holomorphic instantons in the third plane. Finally, for $\theta^{2} \theta^{2} \theta \theta$, we have (i) holomorphic instantons in the first plane (ii) holomorphic and anti-holomorphic instantons in the second plane.

Notice that, despite the fact that the second plane has an untwisted field in the $\theta^{4} \theta^{4} \theta^{3} \theta$ and $\theta^{3} \theta \theta \theta$ couplings, it turns out that instanton solutions can still play a role in the 4-point functions. Intuitively, we can think of this as being due to the three twisted strings and an untwisted one stretching and interacting to form an intermediate worldsheet instanton state pinned to the three associated fixed points. The same could not be said for the 3point couplings, with two twisted fields and one untwisted field in a given plane, because if the worldsheet is pinned only to two fixed points it will tend to collapse.

With the above information in hand, we can write down the additional selection rules, which apply when there are oscillator states involved. The following conditions must be met if the couplings are to be non-vanishing:

- For $\theta^{4} \theta^{4} \theta^{3} \theta$ : Rule 5 imposes (i) $\mathcal{N}_{L}^{2} \geq \overline{\mathcal{N}}_{L}^{2}$ (ii) $\mathcal{N}_{L}^{3}=\overline{\mathcal{N}}_{L}^{3}$ and $\overline{\mathcal{N}}_{R}^{3}=0$. Moreover, Rule 4 imposes (iii) $\mathcal{N}_{L}^{2}-\overline{\mathcal{N}}_{L}^{2}-\overline{\mathcal{N}}_{R}^{2}=0 \bmod 6$, when all three twisted fields are at the same fixed point in the second plane.

- For $\theta^{3} \theta \theta \theta$ : Rule 5 imposes (i) $\mathcal{N}_{L}^{1} \geq \overline{\mathcal{N}}_{L}^{1}$ (ii) $\mathcal{N}_{L}^{2} \geq \overline{\mathcal{N}}_{L}^{2}$. Moreover, Rule 4 imposes (iii) $\mathcal{N}_{L}^{2}-\overline{\mathcal{N}}_{L}^{2}-\overline{\mathcal{N}}_{R}^{2}=0 \bmod 6$, when all twisted fields are at the same fixed point in the second plane (iv) $\mathcal{N}_{L}^{3}-\overline{\mathcal{N}}_{L}^{3}-\overline{\mathcal{N}}_{R}^{3}=0 \bmod 4$, when all twisted fields are at the same fixed point in the third plane.

- For $\theta^{2} \theta^{2} \theta \theta$ : Rule 5 imposes (i) $\mathcal{N}_{L}^{1} \geq \overline{\mathcal{N}}_{L}^{1}$ (ii) $\mathcal{N}_{L}^{3}=\overline{\mathcal{N}}_{L}^{3}$ and $\overline{\mathcal{N}}_{R}^{3}=0$. Moreover, Rule 4 imposes (iii) $\mathcal{N}_{L}^{2}-\overline{\mathcal{N}}_{L}^{2}-\overline{\mathcal{N}}_{R}^{2}=0 \bmod 6$, when all fields are at the same fixed point in the second plane.

\subsection{General L-point couplings}

Let us write a general L-point coupling as $(\theta)^{l_{1}}\left(\theta^{2}\right)^{l_{2}}\left(\theta^{3}\right)^{l_{3}}\left(\theta^{4}\right)^{l_{4}}$ with integers $l_{1,2,3,4} \geq 0$ and $l_{1}+l_{2}+l_{3}+l_{4}=\mathrm{L}$. 
We first show that the H-momentum is automatically conserved, once the point group selection rule and R-charge conservation have been imposed. The point group selection rule constrains the twisted sectors to obey $l_{1}+2 l_{2}+3 l_{3}+4 l_{4}=6 \mathrm{~m}$, with $\mathrm{m}$ a non-zero natural number. Meanwhile, using the H-momenta for the various twisted sectors given at the beginning of this section, we can write the H-momentum conservation condition in each plane as:

$$
\begin{aligned}
\frac{1}{6}\left(l_{1}+2 l_{2}+3 l_{3}+4 l_{4}\right) & =1+\overline{\mathcal{N}}_{R}^{1}, \\
\frac{1}{3}\left(l_{1}+2 l_{2}+l_{4}\right) & =1+\overline{\mathcal{N}}_{R}^{2}, \\
\frac{1}{2}\left(l_{1}+l_{3}\right) & =1+\overline{\mathcal{N}}_{R}^{3},
\end{aligned}
$$

with integers $\overline{\mathcal{N}}_{R}^{i} \geq 0$. We have to check that these conditions can all be satisfied for appropriate choices of $\overline{\mathcal{N}}_{R}^{i}$. On applying the point group selection rule, the first condition can immediately be satisfied. The second condition requires that $l_{1}+2 l_{2}+l_{4}=3 \mathrm{~m}$ with $\mathrm{m}$ a non-zero natural number, and again the point group selection rules ensures this, unless $l_{1,2,4}=0$. Similarly, the third condition can be rewritten as $l_{1}+l_{3}=2 \mathrm{~m}$, which is ensured by the point group selection rule, unless $l_{1,3}=0$. Note, as we saw already in the previous section, the sum of the H-momentum conditions over all planes gives $\sum_{i} \overline{\mathcal{N}}_{R}^{i}=\mathrm{L}-3$.

Consider now the case where the H-momentum conservation cannot be satisfied because $l_{1,2,4}=0$. Since all the fields are then in the third twisted sector, they are all untwisted in the second plane. The masslessness condition then ensures that these modes have no oscillators in the second plane, so that $\mathrm{R}_{\alpha}^{2}=q_{\mathrm{sh} \alpha}^{2}=0$ and $\sum_{\alpha} \mathrm{R}_{\alpha}^{2}=0$. These couplings are then excluded by the R-charge conservation law. Similarly, when $l_{1,3}=0$, all fields are untwisted in the third plane, and it follows that the coupling is excluded by R-conservation in that plane.

Similarly, we can study the H-momentum conservation for higher order couplings allowed by the standard selection rules in (factorizable) $T^{6} / \mathbb{Z}_{3}, T^{6} / \mathbb{Z}_{4}$, and $T^{6} / \mathbb{Z}_{6-I}$ orbifolds.

Finally, we are ready to write down an algorithm to compute when general L-point couplings are allowed. We recall here that we always consider the positive-chiral states.

1. Apply the standard selection rules; gauge invariance, R-charge conservation and the space group selection rule.

2. Use H-momentum conservation to compute how the right-moving oscillators are distributed amongst the three complex planes, $\overline{\mathcal{N}}_{R}^{i}=\sum_{\alpha} q_{\text {sh } \alpha}^{i}-1$.

3. Apply Rule 5. Check whether holomorphic and anti-holomorphic instantons in the $i$-th plane are allowed. For non-trivial holomorphic solutions to exist we require $1+\sum_{\alpha}\left(-1+\mathbf{k}_{\alpha}^{i}\right)<0$ (where $\left.0<\mathbf{k}_{\alpha}^{i} \leq 1\right)$. For non-trivial anti-holomorphic solutions to exist we require $1+\sum_{\alpha}\left(-\mathbf{k}_{\alpha}^{i}\right)<0$ (where $0 \leq \mathbf{k}_{\alpha}^{i}<1$ ). If there are neither holomorphic nor anti-holomorphic solutions, then we require $\overline{\mathcal{N}}_{R}^{i}=0$ and $\mathcal{N}_{L}^{i}=\overline{\mathcal{N}}_{L}^{i}$. If holomorphic instantons are allowed, but anti-holomorphic instantons are forbidden, 


\begin{tabular}{|c|c|c|c|c|}
\hline & \multicolumn{2}{|c|}{ Model 1 } & \multicolumn{2}{c|}{ Model 2 } \\
order & no rules 4 \& 5 & with rules 4 \& 5 & no rules 4 \& 5 & with rules 4 \& 5 \\
\hline 3 & 116 & 112 & 160 & 152 \\
4 & 144 & 142 & 300 & 282 \\
5 & 1446 & $1207(+112)$ & 4710 & $4435(+152)$ \\
6 & 12544 & $8435(+142)$ & 55638 & $49898(+282)$ \\
7 & 108796 & $96548(+1319)$ & 862893 & $833641(+4587)$ \\
\hline
\end{tabular}

Table 1. Number of admissible couplings at different orders for the $\mathbb{Z}_{6-I I}$ MSSM candidates studied in [48]. In the second and fourth columns, we have considered only the standard selection rules discussed in section 2.2. In the third and fifth columns we count the number of couplings that satisfy additionally the new selection rules 4 and 5 . In the parentheses, we add the effective couplings that are generated by holomorphic matter terms in the Kähler potential once the moduli are integrated out, as discussed in section 3.5.

then $\mathcal{N}_{L}^{i} \geq \overline{\mathcal{N}}_{L}^{i}$. If instead only anti-holomorphic instantons are allowed, then $\overline{\mathcal{N}}_{R}^{i}=0$ and $\mathcal{N}_{L}^{i} \leq \overline{\mathcal{N}}_{L}^{i}$.

4. Apply Rule 4 in the second plane: if all twisted fields are at the same fixed point in the second plane, then Rule 4 imposes $\mathcal{N}_{L}^{2}-\overline{\mathcal{N}}_{L}^{2}-\overline{\mathcal{N}}_{R}^{2}=0 \bmod 6$.

5. Apply Rule 4 in the third plane: if all twisted fields are at the same fixed point in the third plane, then Rule 4 imposes $\mathcal{N}_{L}^{3}-\overline{\mathcal{N}}_{L}^{3}-\overline{\mathcal{N}}_{R}^{3}=0 \bmod 4$.

\subsection{Explicit $\mathbb{Z}_{6-I I}$ MSSM candidates}

In order to study the phenomenological consequences of the new selection rules, we proceed now to apply the previous results to identify the allowed couplings in the two MSSM candidates studied in [48] (the defining parameters and matter spectra are provided in that reference). We identify the admissible couplings between all matter states of the models up to order 7 in the superpotential, first ignoring the new selection rules and then taking them into account. In this way, we also confirm that each of the rules has a nontrivial effect and eliminate couplings. The number of allowed couplings before and after applying the new selection rules is provided in table 1 .

We find that around $10 \%$ of the couplings that satisfy the standard selection rules vanish after imposing the new rules. It is then natural to address the phenomenological impact that this reduction of non-vanishing interactions might have. With this aim, we have computed the mass matrices of exotic matter in supersymmetric vacuum configurations considering only the standard rules and including the new rules. The mass matrices are (almost) identical in both cases and all exotics decouple. We expect that this behaviour is reproduced in all models of the Mini-Landscape [3-6].

A positive phenomenological effect of the new rules appears in the proton-decay sector. Proton decay is produced by the simultaneous presence of the effective operators $Q_{i} Q_{j} Q_{k} L_{l}$ and $\bar{u}_{i} \bar{u}_{j} \bar{d}_{k} \bar{e}_{l}$ (where the supermultiplets $Q_{i}$ denote quark doublets, $\bar{u}_{i}, \bar{d}$ are up and downtype quark singlets, and $L_{i}$ represent lepton doublets). These dangerous operators appear 
frequently in the effective theories of $\mathbb{Z}_{6-I I}$ orbifolds. We find that between $15 \%$ and $30 \%$ of these couplings turn out to vanish, once the new selection rules are applied.

Let us make a final remark. The Mini-Landscape models ${ }^{12}$ used in table 1 have one pair of vector-like untwisted matter fields associated to the $\mathbb{Z}_{2}$ plane. As discussed in section 3.5, these would provide effective holomorphic matter couplings once the Kähler and complex structure moduli have been integrated out, which we have counted in the parentheses of table 1. Such couplings could help to address some phenomenological issues [49].

\section{Discussion}

When building realistic models from string theory, one of the most essential aspects to understand are the couplings within the low energy effective field theory. The first question to ask is which couplings are non-vanishing, and the answer can be found by applying string coupling selection rules, which are derived from the structure of the corresponding correlation functions. The correlation functions of interest are tree-level L-point $\left\langle V_{F} V_{F} V_{B} \ldots V_{B}\right\rangle$, since these correspond to superpotential couplings in the low energy effective field theory. The rules thus derived can often be understood in terms of symmetries and charge conservation in the effective field theory.

In heterotic orbifold compactifications, the rules usually considered are gauge invariance, R-charge conservation and the space group selection rule. In this paper we show that there are two additional selection rules, which further restrict the allowed couplings in the superpotential. These rules are both relevant when the candidate couplings between twisted fields involve oscillators, which are couplings between excited states and higher order couplings.

Rule 4 was introduced in the literature in $[11,19]$ but has not been applied in recent works. It is important when the symmetries of the torus lattice, $\mathbb{Z}_{\mathrm{K}^{i}}$ in a plane $i$, are larger than the point group $\mathbb{Z}_{N^{i}}$. When all the twisted fields are at the same fixed point, this additional symmetry is observed in the sum over instanton solutions that mediate the coupling. Then, further to the twist invariance $\mathcal{N}_{L}^{i}-\overline{\mathcal{N}}_{L}^{i}-\overline{\mathcal{N}}_{R}^{i}=0 \bmod \mathrm{N}^{i}$, this leads to $\mathcal{N}_{L}^{i}-\overline{\mathcal{N}}_{L}^{i}-\overline{\mathcal{N}}_{R}^{i}=0 \bmod \mathrm{K}^{i}$ for all twisted fields at the same fixed point. Notice, we might also choose to write Rule 4 in terms of the picture-independent R-charges: $\sum_{\alpha} \mathrm{R}_{\alpha}^{i}=1 \bmod \mathrm{K}^{i}$ for all twisted fields at the same fixed point. Take care, however, that whereas Rule 4 corresponds to the Lorentz symmetries that would survive the toroidal compactification, the actual R-symmetry in the low energy effective field theory of course corresponds to the Lorentz symmetries that survive the full orbifold compactification. Indeed, since Rule 4 also depends on the relative distance between the twisted fields in the compact dimensions, it cannot be simply understood as an R-charge conservation in the low energy effective field theory [20]. Another interesting observation is that the rule eliminates couplings between twisted fields at the same fixed point, which is precisely when we might expect them to be able to interact at order one, field theoretically. This is somehow

\footnotetext{
${ }^{12}$ We have verified that all Mini-Landscape models with two Wilson lines have one pair of vector-like untwisted matter fields. All but seven Mini-Landscape models with three Wilson lines have one or more vector-like pairs.
} 
similar to the phenomenon that an allowed coupling between twisted fields at the same fixed point may be exponentially suppressed if it involves oscillators.

Rule 5 is another stringy rule, which has not appeared before in the literature. It arises when the local monodromy conditions and the required convergence properties for the holomorphic and/or antiholomorphic classical solutions, in a given plane $i$, are satisfied only by the trivial solutions, so that worldsheet instanton solutions are not available there to mediate the couplings. Quantum effects or/and allowed instantons may be sufficient to ensure non-vanishing couplings, but not if the correlation function is proportional to the classical solutions $\partial X_{\mathrm{cl}}^{i}$ and/or $\partial \bar{X}_{\mathrm{cl}}^{i}$, or their complex conjugates. This leads to conditions such as $\mathcal{N}_{L}^{i} \geq \overline{\mathcal{N}}_{L}^{i}$ if only holomorphic instantons are allowed in the plane $i$.

We close with a few more observations. When considering the rules for higher order couplings, a natural question is what are the consequences of picture-changing. For an L-point coupling of kind $\psi \psi \phi^{\mathrm{L}-2}$, ghost-charge cancellation requires that $\mathrm{L}-3$ picturechanging operators are introduced into the correlation function. Invariance under picturechanging manifests itself in the fact that the physics — including the rules - is invariant under how we choose to distribute the picture-changing amongst the fields [12]. Note that this seems to make it difficult to express Rule 5 in terms of possible charges carried by each of the participating fields in a coupling; instead the rule depends explicitly on the total number of right-moving oscillators introduced into the correlation function by picture-changing.

Indeed, it remains an important open question whether Rules 4 and 5 correspond to conventional global and/or local symmetries in the low energy effective field theory, or instead represent intriguing stringy miracles. We should point out that Rule 5 has not been understood in terms of a symmetry, even at the stringy level. This is similar to the space group selection rule, which is due to the boundary conditions of the interacting strings, although the latter can at least be partially understood in terms of various $\mathbb{Z}_{N}$-type global symmetries $[2,8,21]$.

In many models, R-symmetries and other discrete symmetries are anomalous [50]. It would be important to study how those anomalies affect Rule 4 as well as Rule 5. We will study these aspects elsewhere.

Another interesting problem is the extension of the rules to the case of non-factorizable orbifolds. Those include $T^{6} / \mathbb{Z}_{7}, T^{6} / \mathbb{Z}_{8-I}, T^{6} / \mathbb{Z}_{8-I I}, T^{6} / \mathbb{Z}_{12-I}$ and $T^{6} / \mathbb{Z}_{12-I I}$ orbifolds [16, 28]. In addition, even $T^{6} / \mathbb{Z}_{3}, T^{6} / \mathbb{Z}_{4}, T^{6} / \mathbb{Z}_{6-I}$ and $T^{6} / \mathbb{Z}_{6-I I}$ orbifolds as well as $T^{6} /\left(\mathbb{Z}_{M} \times \mathbb{Z}_{N}\right)$ orbifolds are also realized as non-factorizable orbifolds [16, 28, 34, 51-54]. In fact, even the R-charge conservation law has not yet been understood for non-factorizable orbifolds [27].

Our focus in the present paper has been in selection rules for superpotential couplings. The ultimate objective would be a derivation of the full low energy effective field theory describing the orbifold compactification, in terms of the superpotential, Kähler potential, gauge kinetic functions and Fayet-Iliopolous terms. Much less is known about the Kähler potential, but it can be derived by computing four boson scattering amplitudes between matter fields and moduli fields [55], and can include some contributions where all the matter fields are holomorphic. After moduli stabilization, such terms would lead to new couplings 
in an effective superpotential, relevant for phenomenology. Thus it would be essential to study further the Kähler potential.

Finally, we have briefly illustrated the implementation of the new rules in some $\mathbb{Z}_{6-I I}$ orbifold MSSM candidates, including the known holomorphic matter contributions to the Kähler potential. Couplings are crucial to understanding the dynamics of such models, for instance the decoupling of exotics, as well as for example the quark and lepton masses. Our initial results indicate that the heterotic orbifold Mini-Landscape [3-6] continues to provide promising phenomenological models. The new selection rules must now be implemented in all such orbifold studies.

\section{Acknowledgments}

We thank N. Cabo-Bizet, O. Lebedev, C. Lüdeling, D. Mayorga-Peña, H.P. Nilles, N. Pagani, M. Ratz and G. Tasinato for helpful discussions. T.K. is supported in part by the Grant-in-Aid for Scientific Research No. 20540266 and the Grant-in-Aid for the Global COE Program "The Next Generation of Physics, Spun from Universality and Emergence" from the Ministry of Education, Culture, Sports, Science and Technology of Japan. S.L.P. is supported by the Göran Gustafsson Foundation. I.Z. was supported by the DFG cluster of excellence Origin and Structure of the Universe, the SFB-Tansregio TR33 "The Dark Universe" (Deutsche Forschungsgemeinschaft) and the European Union 7th network program "Unification in the LHC era" (PITN-GA-2009-237920). S.R.-S. was partially supported by CONACyT project 82291 and DGAPA project IA101811.

\section{References}

[1] W. Buchmüller, K. Hamaguchi, O. Lebedev and M. Ratz, Supersymmetric standard model from the heterotic string, Phys. Rev. Lett. 96 (2006) 121602 [hep-ph/0511035] [INSPIRE].

[2] W. Buchmüller, K. Hamaguchi, O. Lebedev and M. Ratz, Supersymmetric standard model from the heterotic string (II), Nucl. Phys. B 785 (2007) 149 [hep-th/0606187] [INSPIRE].

[3] O. Lebedev et al., A mini-landscape of exact MSSM spectra in heterotic orbifolds, Phys. Lett. B 645 (2007) 88 [hep-th/0611095] [INSPIRE].

[4] O. Lebedev et al., The heterotic road to the MSSM with R parity, Phys. Rev. D 77 (2008) 046013 [arXiv:0708.2691] [INSPIRE].

[5] O. Lebedev, H.P. Nilles, S. Ramos-Sánchez, M. Ratz and P.K.S. Vaudrevange, Heterotic mini-landscape. (II): Completing the search for MSSM vacua in a $Z_{6}$ orbifold, Phys. Lett. B 668 (2008) 331 [arXiv:0807.4384] [INSPIRE].

[6] M. Blaszczyk et al., $A Z_{2} \times Z_{2}$ standard model, Phys. Lett. B 683 (2010) 340 [arXiv:0911.4905] [INSPIRE].

[7] T. Kobayashi, S. Raby and R.-J. Zhang, Constructing 5-D orbifold grand unified theories from heterotic strings, Phys. Lett. B 593 (2004) 262 [hep-ph/0403065] [INSPIRE].

[8] T. Kobayashi, S. Raby and R.-J. Zhang, Searching for realistic 4d string models with a Pati-Salam symmetry: orbifold grand unified theories from heterotic string compactification on a $Z_{6}$ orbifold, Nucl. Phys. B 704 (2005) 3 [hep-ph/0409098] [INSPIRE]. 
[9] J.E. Kim and B. Kyae, Flipped $\mathrm{SU}(5)$ from $Z_{12-I}$ orbifold with Wilson line, Nucl. Phys. B 770 (2007) 47 [hep-th/0608086] [INSPIRE].

[10] J.E. Kim, J.-H. Kim and B. Kyae, Superstring standard model from $Z_{12-I}$ orbifold compactification with and without exotics and effective R-parity, JHEP 06 (2007) 034 [hep-ph/0702278] [INSPIRE].

[11] S. Hamidi and C. Vafa, Interactions on orbifolds, Nucl. Phys. B 279 (1987) 465 [InSPIRE].

[12] L.J. Dixon, D. Friedan, E.J. Martinec and S.H. Shenker, The conformal field theory of orbifolds, Nucl. Phys. B 282 (1987) 13 [INSPIRE].

[13] T.T. Burwick, R.K. Kaiser and H.F. Müller, General Yukawa couplings of strings on $Z_{N}$ orbifolds, Nucl. Phys. B 355 (1991) 689 [INSPIRE].

[14] J. Erler, D. Jungnickel, M. Spalinski and S. Stieberger, Higher twisted sector couplings of $Z_{N}$ orbifolds, Nucl. Phys. B 397 (1993) 379 [hep-th/9207049] [INSPIRE].

[15] T. Kobayashi and N. Ohtsubo, Yukawa coupling condition of $Z_{N}$ orbifold models, Phys. Lett. B 245 (1990) 441 [inSPIRE].

[16] T. Kobayashi and N. Ohtsubo, Geometrical aspects of $Z_{N}$ orbifold phenomenology, Int. J. Mod. Phys. A 9 (1994) 87 [inSPIRE].

[17] D. Bailin, A. Love and W.A. Sabra, Yukawa couplings involving excited twisted sector states for $Z_{N}$ and $Z_{M} \times Z_{N}$ orbifolds, Nucl. Phys. B 416 (1994) 539 [hep-th/9307172] [INSPIRE].

[18] K.-S. Choi and T. Kobayashi, Higher order couplings from heterotic orbifold theory, Nucl. Phys. B 797 (2008) 295 [arXiv:0711.4894] [INSPIRE].

[19] A. Font, L.E. Ibáñez, H.P. Nilles and F. Quevedo, Degenerate orbifolds, Nucl. Phys. B 307 (1988) 109 [Erratum ibid. B 310 (1988) 764] [INSPIRE].

[20] A. Font, L.E. Ibáñez, H.P. Nilles and F. Quevedo, On the concept of naturalness in string theories, Phys. Lett. B 213 (1988) 274 [INSPIRE].

[21] J. Schmidt, Local grand unification in the heterotic landscape, Fortschr. Phys. 58 (2010) 3 [arXiv:0906.5501] [INSPIRE].

[22] T. Kobayashi, H.P. Nilles, F. Plöger, S. Raby and M. Ratz, Stringy origin of non-Abelian discrete flavor symmetries, Nucl. Phys. B 768 (2007) 135 [hep-ph/0611020] [INSPIRE].

[23] P. Ko, T. Kobayashi, J.-h. Park and S. Raby, String-derived $D_{4}$ flavor symmetry and phenomenological implications, Phys. Rev. D 76 (2007) 035005 [Erratum ibid. D 76 (2007) 059901] [arXiv: 0704.2807] [INSPIRE].

[24] D. Bailin and A. Love, Orbifold compactifications of string theory, Phys. Rept. 315 (1999) 285 [INSPIRE].

[25] K.-S. Choi and J.E. Kim, Quarks and leptons from orbifolded superstring, Lect. Notes Phys. 696 (2006) 1 [INSPIRE].

[26] P.K.S. Vaudrevange, Grand unification in the heterotic brane world, arXiv:0812.3503 [INSPIRE].

[27] S. Ramos-Sánchez, Towards low energy physics from the heterotic string, Fortschr. Phys. 10 (2009) 907 [arXiv:0812.3560] [INSPIRE].

[28] Y. Katsuki et al., $Z_{N}$ orbifold models, Nucl. Phys. B 341 (1990) 611 [InSPIRE]. 
[29] P. Goddard and D.I. Olive, Kac-Moody and Virasoro algebras in relation to quantum physics, Int. J. Mod. Phys. A 1 (1986) 303 [inSPIRE].

[30] M. Cvetič, Suppression of nonrenormalizable terms in the effective superpotential for (blownup) orbifold compactification, Phys. Rev. Lett. 59 (1987) 1795 [INSPIRE].

[31] J. Polchinski, String theory. Vol. 2: Superstring theory and beyond, Cambridge University Press, Cambridge U.K. (1998) [INSPIRE].

[32] M. Dine and N. Seiberg, Nonrenormalization theorems in superstring theory, Phys. Rev. Lett. 57 (1986) 2625 [INSPIRE].

[33] L.E. Ibáñez, J. Mas, H.-P. Nilles and F. Quevedo, Heterotic strings in symmetric and asymmetric orbifold backgrounds, Nucl. Phys. B 301 (1988) 157 [INSPIRE].

[34] F. Plöger, S. Ramos-Sánchez, M. Ratz and P.K.S. Vaudrevange, Mirage torsion, JHEP 04 (2007) 063 [hep-th/0702176] [INSPIRE].

[35] M. Bershadsky and A. Radul, Conformal field theories with additional $Z_{N}$ symmetry, Int. J. Mod. Phys. A 2 (1987) 165 [inSPIRE].

[36] J.J. Atick, L.J. Dixon, P.A. Griffin and D. Nemeschansky, Multiloop twist field correlation functions for $Z_{N}$ orbifolds, Nucl. Phys. B 298 (1988) 1 [INSPIRE].

[37] S.A. Abel and A.W. Owen, $N$ point amplitudes in intersecting brane models, Nucl. Phys. B 682 (2004) 183 [hep-th/0310257] [InSPIRE].

[38] T. Kobayashi, Selection rules for nonrenormalizable couplings in superstring theories, Phys. Lett. B 354 (1995) 264 [hep-ph/9504371] [INSPIRE].

[39] A. Font, L.E. Ibáñez, H.P. Nilles and F. Quevedo, Yukawa couplings in degenerate orbifolds: towards a realistic $\mathrm{SU}(3) \times \mathrm{SU}(2) \times \mathrm{U}(1)$ superstring, Phys. Lett. 210B (1988) 101 [Erratum ibid. B 213 (1988) 564] [INSPIRE].

[40] A. Font, L.E. Ibáñez, F. Quevedo and A. Sierra, The construction of 'realistic' four-dimensional strings through orbifolds, Nucl. Phys. B 331 (1990) 421 [INSPIRE].

[41] P. Mayr and S. Stieberger, Low-energy properties of (0,2) compactifications, hep-th/9412196 [INSPIRE].

[42] T. Kobayashi and O. Lebedev, Heterotic Yukawa couplings and continuous Wilson lines, Phys. Lett. B 566 (2003) 164 [hep-th/0303009] [INSPIRE].

[43] G. Lopes Cardoso, D. Lüst and T. Mohaupt, Moduli spaces and target space duality symmetries in (0,2) $Z_{N}$ orbifold theories with continuous Wilson lines, Nucl. Phys. B 432 (1994) 68 [hep-th/9405002] [INSPIRE].

[44] I. Antoniadis, E. Gava, K.S. Narain and T.R. Taylor, Effective $\mu$ term in superstring theory, Nucl. Phys. B 432 (1994) 187 [hep-th/9405024] [INSPIRE].

[45] M. Cvetič, B.A. Ovrut and W. Sabra, Kähler potentials for orbifolds with continuous Wilson lines and the symmetries of the string action, Phys. Lett. B 351 (1995) 173 [hep-th/9502144] [INSPIRE].

[46] A. Brignole, L.E. Ibáñez and C. Muñoz, Orbifold induced mu term and electroweak symmetry breaking, Phys. Lett. B 387 (1996) 769 [hep-ph/9607405] [INSPIRE].

[47] F. Brümmer, R. Kappl, M. Ratz and K. Schmidt-Hoberg, Approximate R-symmetries and the $\mu$ term, JHEP 04 (2010) 006 [arXiv: 1003.0084] [INSPIRE]. 
[48] S.L. Parameswaran, S. Ramos-Sánchez and I. Zavala, On moduli stabilisation and de Sitter vacua in MSSM heterotic orbifolds, JHEP 01 (2011) 071 [arXiv: 1009.3931] [INSPIRE].

[49] R. Kappl et al., Large hierarchies from approximate $R$ symmetries, Phys. Rev. Lett. 102 (2009) 121602 [arXiv:0812.2120] [INSPIRE].

[50] T. Araki et al., (Non-)Abelian discrete anomalies, Nucl. Phys. B 805 (2008) 124 [arXiv: 0805.0207] [INSPIRE].

[51] A.E. Faraggi, S. Förste and C. Timirgaziu, $Z_{2} \times Z_{2}$ heterotic orbifold models of non factorisable six dimensional toroidal manifolds, JHEP 08 (2006) 057 [hep-th/0605117] [INSPIRE].

[52] S. Förste, T. Kobayashi, H. Ohki and K.-J. Takahashi, Non-factorisable $Z_{2} \times Z_{2}$ heterotic orbifold models and Yukawa couplings, JHEP 03 (2007) 011 [hep-th/0612044] [INSPIRE].

[53] K.-J. Takahashi, Heterotic orbifold models on Lie lattice with discrete torsion, JHEP 03 (2007) 103 [hep-th/0702025] [INSPIRE].

[54] K.-J. Takahashi, Three-family GUT models from heterotic orbifold on $E_{6}$ root lattice, Prog. Theor. Phys. 119 (2008) 491 [arXiv:0707.3355] [INSPIRE].

[55] L.J. Dixon, V. Kaplunovsky and J. Louis, On effective field theories describing (2,2) vacua of the heterotic string, Nucl. Phys. B 329 (1990) 27 [INSPIRE]. 